\title{
Clave para la determinación de exuvias pupales de los quironómidos (Diptera: Chironomidae) de ríos altoandinos tropicales
}

\author{
Narcís Prat ${ }^{1 *}$, Juan David González-Trujillo² \& Rodulfo Ospina-Torres ${ }^{2}$ \\ 1. Grupo de Investigación F.E.M. (Freshwater Ecology and Management), Universidad de Barcelona, Barcelona, \\ España; nprat@ub.edu \\ 2. Laboratorio de Invertebrados Acuáticos, Departamento de Biología, Universidad Nacional de Colombia, Bogotá, \\ Colombia; jdgonzalezt@unal.edu.co,rospinat@unal.edu.co \\ * Correspondencia
}

Recibido 15-V-2013. Corregido 01-V-2014. Aceptado 06-VI-2014.

\begin{abstract}
Key to chironomid pupal exuviae (Diptera: Chironomidae) of tropical high Andean streams. The Chironomidae is a cosmopolitan family of Nematoceran flies with more than 20000 species described. However the diversity of genera and species of the family in the Andean region beyond the 2000m.a.s.1 are scarcely known. We conducted faunal surveys and biomonitoring research in different streams of Colombia, Ecuador and Peru from May 2005 to October 2011. Based on specimens collections, a taxonomic key was developed to identify pupae and pupal exuviae of 46 genera of midges (Diptera, Chironomidae) collected from streams at high altitude areas on the Andean tropical mountains. We included illustrations and brief taxonomic descriptions for all genera, of which several ones have not yet been formally described; in this latter case we used the nomenclature of Roback \& Coffman (1983). For two genera, Cricotopus and Genus 1, keys to the most common morphospecies were provided. Results showed that in this area the chironomid assemblages are dominated by the members of the subfamily Orthocladiinae (22 genera) followed by the Chironominae (13). Six genera of Tanypodinae were identified, while only three and two genera were present from subfamilies Podonominae and Diamesinae. This key may be very useful for both studies about drift in streams, and for biomonitoring purposes. Rev. Biol. Trop. 62 (4): 1385-1406. Epub 2014 December 01.
\end{abstract}

Key words: Chironomidae, high Andean streams, pupae, pupal exuviae, drift.

La zona altoandina tropical comprende aquellos sectores del norte de los Andes (desde el sur de Perú hasta el norte de Colombia) que se encuentran ubicados por encima de los $2000 \mathrm{msnm}$. En esta región biogeográfica que comprende la cordillera andina, la ausencia o diminución de las estaciones climáticas se combina con las condiciones propias de la alta montaña (p.e. clima frío) y se traduce en una disminución de la biodiversidad de los macroinvertebrados acuáticos con la altitud (Jacobsen, 2008). Por su situación geográfica, esta zona tiene una gran biodiversidad, y se pueden encontrar taxones de origen Austral, Holártico y algunos que se han originado localmente, tal como se ha visto con algunas formaciones vegetales (Weigend, Gottschling, Higler \& Nuck, 2010). Es de esperar que esta situación también se de en los dípteros de la familia Chironomidae que habitan en los ríos tropicales altoandinos y de aquí el interés de profundizar en su conocimiento.

Las larvas de dípteros de la familia Chironomidae son muy abundantes en los ríos de todo el mundo, conformando muchas veces una buena parte de las comunidades de macroinvertebrados acuáticos, así como de la biomasa animal de estos ecosistemas de la que se alimentan otros organismos, entre ellos los peces. A pesar de esto, todavía su taxonomía es casi desconocida en algunos lugares del mundo y muy particularmente en el Neotrópico, en 
donde la mayoría de trabajos están centrados en zonas de baja altitud (Spies \& Reiss, 1996; Wiedenbrug \& Ospina-Torres, 2005). Para la zona tropical altoandina eran muy escasos los trabajos publicados sobre este grupo (Roback, 1970; Roback \& Coffman, 1983). Sin embargo, el interés actual por el estudio de los quironómidos en la zona Neotropical ha propiciado la realización de revisiones taxonómicas que incluyen en ellas algunas especies presentes en la zona altoandina, como los trabajos de la tribu Coryneurinii (Wiedenbrug \& TrivinoStrixino, 2009, 2011; Wiedenbrug, Mendes, Pepinelli, \& Trivino-Strixino, 2009), o de las especies de origen Gondwánico, tanto de la subfamilia Podonominae (Cranston, Hardy, Morse, Pusledik, \& McCluen, 2010) como de la Orthocladiinae (Kros, Baker, \& Cranston, 2011; Prat, Ribera, Rieradevall, Villamarin, \& Acosta, 2013). Destacan a su vez trabajos que aumentan el conocimiento taxonómico del grupo con la descripción de nuevos géneros y especies como en el caso de Oliveiriella (Tejerina \& Paggi, 2009; Wiedenbrug \& Fittkau, 1997) y Barbadocladius (Cranston \& Krosh, 2011; Prat et al., 2013), la revisión de géneros como Stictocladius (Saether \& Cranston, 2012) y la redescripción de especies como Riethia trucatocaudata (Trivino-Strixino, Roque, \& Cranston, 2009). Esta serie de trabajos junto a los estudios realizados por los autores en la zona (Acosta 2009; Acosta \& Prat, 2010; Ruiz-Moreno, Ospina-Torres, \& Riss, 2000a; Ruiz-Moreno, Ospina-Torres, Gomez-Sierra, \& Riss, 2000b; Villamarín, 2012) han supuesto un avance considerable en el conocimiento de este grupo de dípteros en el Neotrópico.

En la actualidad se dispone de claves que permiten identificar los principales géneros de larvas y de morfotipos larvarios de la Sabana de Bogotá (Ospina-Torres, Riss, \& Ruiz-Moreno, 1999; Ruíz- Moreno et al. 2000a, 2000b) y las de los páramos y bosques de la zona altoandina de Perú y Ecuador (Prat, Acosata, Villamarin, \& Rieradevall, 2011), todos estos trabajos están siendo muy útiles para los estudios de comunidades de quironómidos de los ríos tanto de la Patagonia (García \& Añón-Suarez, 2007) como de la zona norte de los Andes de Argentina (Tejerina \& Molinieri, 2007; Medina, Shreiber, \& Paggi, 2008; Tejerina \& Malizia, 2012) o los ríos altoandinos de Perú (Acosta \& Prat, 2010) o de Ecuador (Kuhn et al., 2011), sin embargo, solo uno de los trabajos citados usa exuvias pupales. ¿Por qué es importante la recolección y clasificación de las pupas y exuvias pupales de los quironómidos?, la razón de su uso es que las pupas y exuvias tienen un conjunto de características que permiten distinguir fácilmente los géneros y las especies dentro de los géneros (a veces incluso mejor que con los adultos). Además cuando la pupa es macho y tiene en su interior la genitalia bien formada, puede dar lugar incluso a la descripción de nuevas especies. En este contexto, el poder disponer de una clave para la clasificación del estadio pupal puede ser un incentivo para que se estudien mejor los quironómidos de los ríos altoandinos. El objetivo del presente trabajo consiste en la elaboración de una clave de exuvias pupales de quironómidos de los ríos altoandinos, en gran parte inspirada por el trabajo de Cranston (2000) en Australia, para contribuir al avance del conocimiento de este grupo tan abundante en los ríos de las zonas altoandinas.

\section{MATERIALES Y MÉTODOS}

La recolección del material proviene de diferentes estudios realizados en la zona altoandina desde el sur del Perú al norte de Colombia. Se han estudiado hasta el momento 18 cuencas hidrográficas tanto de la vertiente atlántica como pacífica de los Andes (Cuadro 1). Mientras que en algunos casos se han visitado una sola vez, en otros se trata de trabajos continuados en un mismo río. La recolección proviene tanto de pupas encontradas en los muestreos bentónicos con redes Surber destinadas al estudio de las comunidades, así como de exuvias pupales recolectadas de muestras de deriva en tres proyectos diferentes, el proyecto CERA, el proyecto FUCARA y el proyecto BIQURA, además de los datos de Acosta, 2009; Villamarín, 2012 y de las recogidas en la Sabana de Bogotá y alrededores por Ospina-Torres et 


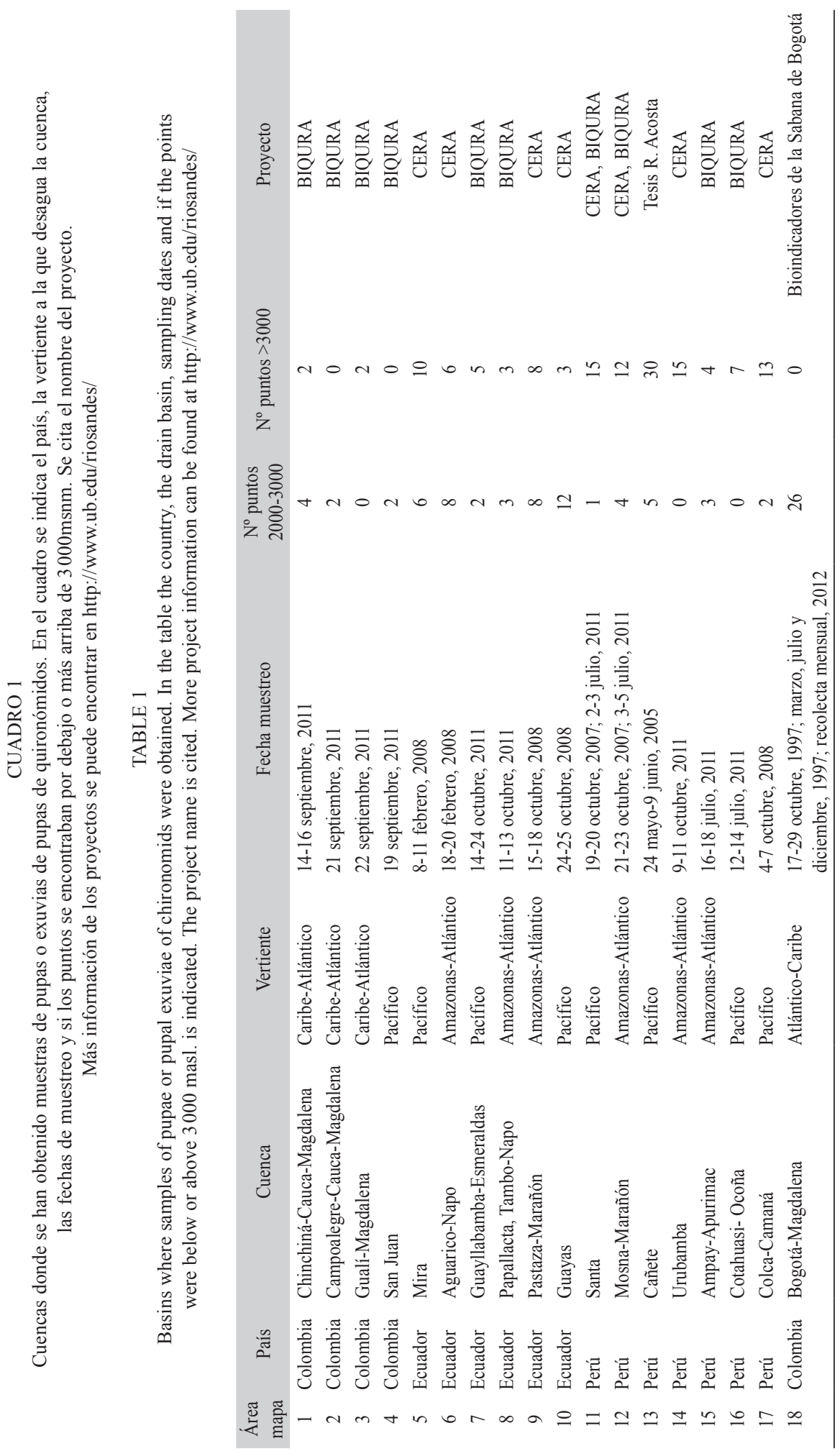


al., 1999 (Tabla 1). Para detalles de los proyectos y sus métodos de recolección véase la página web: http://www.ub.edu/riosandes/ y las publicaciones de Acosta \& Prat (2010), Villamarin, Rieradevall, Paul, Barbour \& Prat (2013) y Prat et al. (2013). En total se efectuaron muestreos en 192 lugares, 115 de ellos situados a más de $3000 \mathrm{msnm}$, y 82 entre los 2000 y los $3000 \mathrm{msnm}$. (Tabla 1). El material recolectado fue fijado en alcohol (70 o 90\% según proyecto) o formol (4\%), y transportado al laboratorio donde se procedió a la separación de las muestras. Las pupas y exuvias fueron contadas y preservadas en alcohol (concentración de $70-96 \%$ dependiendo del proyecto) hasta el momento de su montaje y posterior análisis molecular.

Para la preparación de las muestras se siguió la metodología descrita por Pinder \& Reiss (1986). Las exuvias, al ser habitualmente transparentes no necesitan de un aclarado previo, por lo que se pueden montar directamente, siempre en posición dorsal procurando que los órganos respiratorios queden laterales y fácilmente visibles. En el caso que en los esternitos existan caracteres distintivos, se puede montar alguna exuvia en posición ventral. Las pupas se deben aclarar previamente con una solución de hidróxido de potasio $(\mathrm{KOH})$ caliente. Por lo tal, antes de este procedimiento y según el grado de madurez de las pupas, conviene mirar si la exuvia de la pupa puede separarse del cuerpo del adulto farado si este se halla en su interior, debido a que esto permitiría una mejor observación de ambos estados de desarrollo. Se debe ser especialmente cuidadoso al retirar la exuvia de las pupas de los machos, porque al tener el hipopigio completamente formado es posible asociar la pupa y el adulto a nivel taxonómico de especie. Una vez aclarada la pupa o con la exuvia recogida, se procedió a su preparación microscópica. La mayoría de los ejemplares de nuestras colecciones se encuentran montados en Euparal ${ }^{\circledR}$, lo que implica una deshidratación previa en alcohol del $70 \%$ y $90 \%$ hasta que se procede a su montaje.

Las ilustraciones presentadas en la clave son en su mayoría dibujos basados en fotografías del material preparado por Narcís Prat o dibujos realizados con cámara lúcida a partir de preparaciones, en unos pocos casos son adaptaciones de los dibujos de Coffman (1986). Todos ellos fueron calcados y estandarizados a un único formato mediante el programa Adobe Illustrator ${ }^{\circledR}$ por uno de los autores (GonzálezTrujillo). La morfología de los quironómidos de la clave se basó en las publicaciones de Paggi (2009), Roback \& Coffman (1983) y Wiederholm (1986) (Fig. 1). Para los cuernos torácicos (TH siglas en inglés) se ha preferido emplear el término órgano respiratorio (OR). En el abdomen se distingue entre "ganchos" que son fuertes, curvados dirigidos hacia la zona delantera del abdomen y las "espinas", más finas y dirigidas hacia la parte trasera de los segmentos abdominales. El peine anal del segmento VIII se nombra indistintamente como "espuela" o "espina" según su forma. Si existen sedas abundantes en el segmento anal se considera como una orla de sedas ("fringe" en su término original en inglés).

\section{RESULTADOS}

El resultado principal de este trabajo es la clave que se desarrolla a continuación. En ella se reportan 46 géneros diferentes (5 subfamilias) para los ríos de la zona altoandina. En este trabajo consideramos la zona altoandina la zona de la cordillera de los Andes situada entre el Sur de Perú y el norte de Colombia (ríos altoandinos tropicales) y a altitudes superiores a los $2000 \mathrm{msnm}$. La mayor diversidad la presentó la subfamilia Orthocladiinae con 22 géneros. Para dos de ellos, presentamos una clave preliminar de morfotipos; cinco para el género Cricotopus y ocho para el Género 1 (un género con una combinación única de caracteres en las exuvias pero de los que se desconoce el adulto y por lo tanto su posición taxonómica precisa dentro de los Orthocladiinae). La subfamilia Chironominae la representaron 13 géneros, de los cuales uno pertenece a la tribu Pseudochironominii, cuatro a Tanytarsinii y el resto a Chironominii. De la subfamilia Tanypodinae se reportan seis géneros, mientras que 


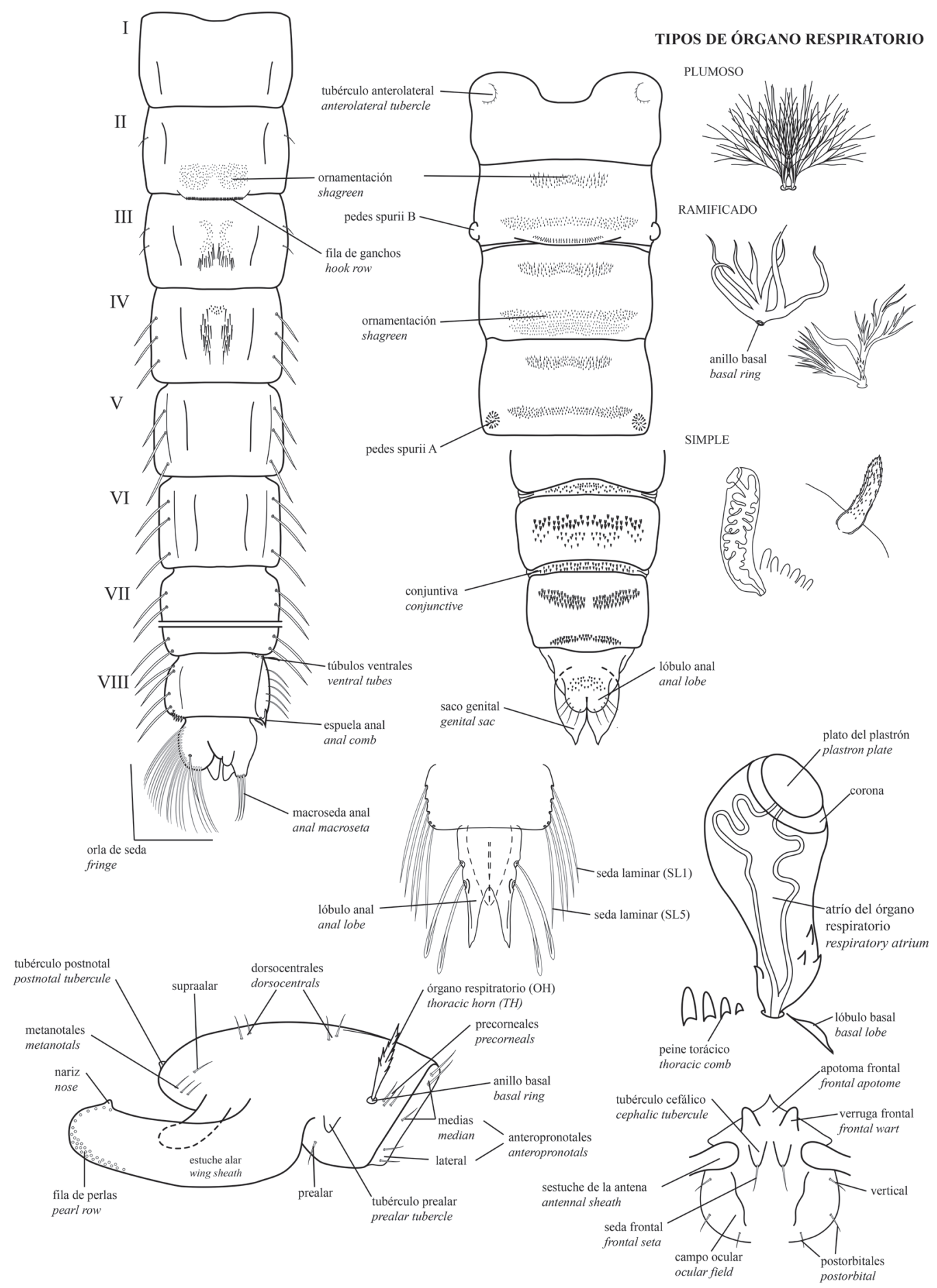

Fig. 1. Morfología de las pupas de quironómidos. La terminología está adaptada de las publicaciones de Coffman (1986) y Paggi (2009).

Fig. 1. Morphology of Chironomidae pupae. Taxonomic terminology was adapted from Coffman (1986) and Paggi (2009). 
para las subfamilias Podonominae y Diamesinae (tribu Heptagyinii) se encontraron tres y dos géneros respectivamente.

De la lista taxonómica, destaca la presencia de algunos géneros considerados como Gondwánicos de acuerdo a Krosh \& Cranston (2011), que en esta zona aparecen confinados a los ríos de mayor altitud (Barbadocladius, Stictocladius). Se constata, sin embargo, la ausencia de muchos otros géneros típicamente gondwánicos que fueron descritos por Brundin (1966) y que han sido citados más tarde sólo de algunos ríos de la Patagonia (García \& AñónSuarez 2007).

\section{CLAVE PARA LAS SUBFAMILIAS (Adaptada de Coffman, 1986)}

1. -Órgano respiratorio (OR) bien desarrollado, menos de la mitad del largo del cefalotórax, con plastrón en posición apical. Lóbulos anales de moderados a bien desarrollados, siempre terminados en punta o con espinas terminales, pero nunca en una orla de sedas. Sus puntas están separadas entre sí. Con sedas onduladas en el segmento abdominal VIII (Fig. 11, Fig. 12 y Fig. 13)

PODONOMINAE -OR bien desarrollado, débilmente desarrollado o ausente, algunas veces ramificados y otras veces con plastrón. Lóbulos anales pueden terminar o no en punta, muy poco frecuente que tengan más de tres macrosedas, aunque pueden estar presentes espinas marginales u orla de sedas. Sin sedas onduladas en el segmento abdominal VIII. . . . . . . . . 2

2. -OR generalmente bien desarrollado, con un plastrón evidente, aunque éste último puede estar poco desarrollado o faltar; pero nunca están ramificados (Fig. 4, Fig. 5, Fig. 6, Fig. 7 y Fig. 9). Lóbulos anales puntiagudos o redondeados, con 2 macrosedas lameliformes o con forma de pelo y rígidas en cada lóbulo; puede presentar orla de sedas o hileras de espinas a lo largo del borde externo e interno del lóbulo..

TANYPODINAE -OR, si están presentes, siempre sin plastrón; a menudo pueden ser ramificados. Lóbulos anales puntiagudos, redondeados o a veces ausentes, nunca con dos sedas lameliformes o en forma de pelo y rígidas, con o sin orla de sedas. 3

3. -Estuches de las patas anteriores y medias hacía atrás, los tarsos de las patas posteriores están cubiertos por los estuches alares. Tergito abdominal II nunca presenta fila de ganchos. Lóbulos anales generalmente sin orla de sedas, pero si está presente es muy corta (Fig. 14, Fig. 15 y Fig. 16) . . . . . . . . . . . . . . . . . DIAMESINAE -Generalmente los estuches de todas las patas está cubiertos por los estuches alares. El tergito abdominal II puede presentar o no una fila de ganchos. Lóbulos anales con o sin orla de sedas, pero cuando está presente las sedas gene-

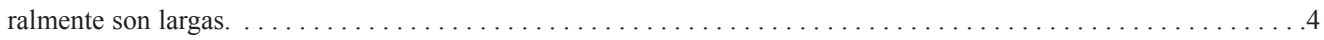

4. -OR nunca ramificado, frecuentemente ausente (Fig. 29, Fig. 38, Fig. 39, Fig. 48). Lóbulos anales con o sin orla de sedas y con o sin macrosedas. Márgenes posterolaterales del segmento VIII casi nunca con un peine anal simple o

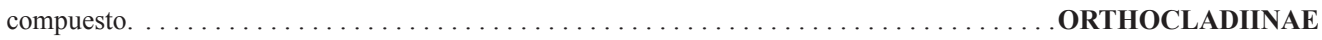
-OR ramificado (Fig. 61, Fig. 62, Fig. 63, Fig. 64, Fig. 65, Fig. 66, Fig. 67, Fig. 68 y Fig. 69), excepto en Tanytarsini que es tubular y algunos Pseudochironomi. Lóbulos anales siempre con orla de sedas, pero nunca con macrosedas. Márgenes posterolaterales del segmento VIII generalmente con un peine anal simple o compuesto. .

CHIRONOMINAE

\section{Subfamilia Tanypodinae}

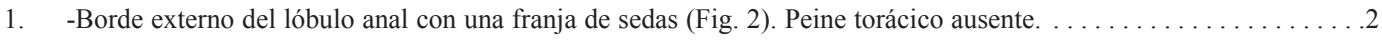

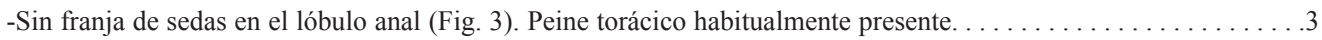

2. -Atrio del OR estrecho, convolvulado; el lumen no lo llena completamente. Superficie de este con un recubrimiento

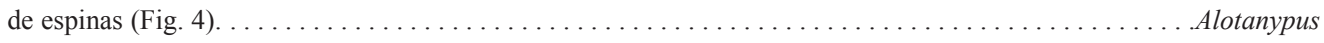
-Atrio del OR grueso, no convolvulado; el lumen lo llena completamente. Superficie de este sin recubrimiento de

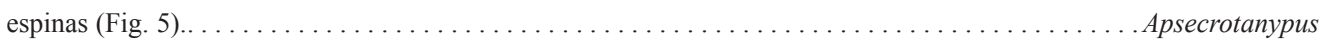

3. -Sin plastrón, OR con un reticulado fino (Fig. 6). Ápice del lóbulo anal puntiagudo y ligeramente

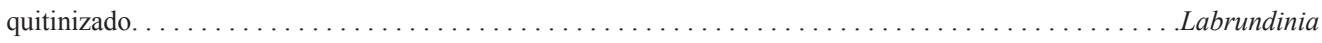
-Plastrón presente en forma de placa.. . . . . . . . . . . . . . . . . . . . . . . . . . . . 4

4. -Plastrón grande, más de la mitad del OR (Fig. 7). Lóbulo anal triangular, no fuertemente puntiagudo y con el ápice portando un pequeño lóbulo adicional (Fig. 3) $\ldots \ldots \ldots \ldots \ldots \ldots \ldots \ldots \ldots \ldots \ldots \ldots \ldots \ldots \ldots \ldots \ldots \ldots \ldots$ 
-Plastrón pequeño, menos de la mitad del OR.

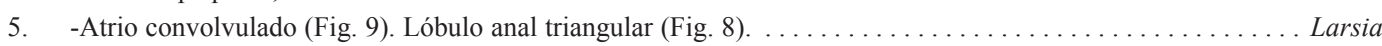

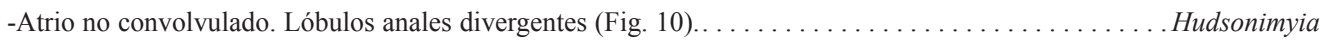

\section{Subfamilia Podonominae}

1. -Segmentos abdominales III-VII prolongados en procesos laterales en el margen posterior (Fig. 12 y Fig. 13) . . . .2

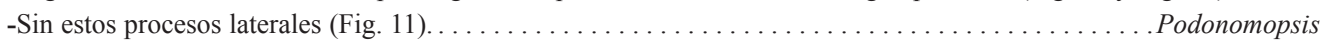

2. -Lóbulos anales como mucho con dos sedas onduladas en cada lado, el mismo segmento tiene dos espinas anales más o menos desarrolladas que tienen 1-3 sedas muy pequeñas (Fig. 12) . . . . . . . . . . . . . . . Parochlus -Lóbulos anales como mínimo con 5 sedas a cada lado. Si hay espinas estas están poco desarrolladas y sin sedas

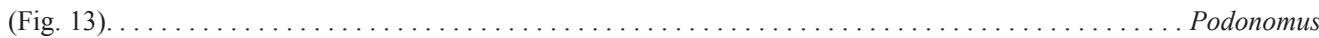

\section{Subfamilia Diamesinae (Tribu Heptagyini)}

1. -Cefalotórax con todas las sedas del tipo normal. Lóbulo anal con 5 sedas a cada lado (Fig. 14). . . . .Paraheptagyia -Cefalotórax como mínimo con una seda precorneal y en algunos casos la seda anteropronotal anterior muy larga y gruesa (Fig. 16). Lóbulo anal sin sedas, como máximo dos sedas a cada lado (Fig. 15)................ Limaya

\section{Subfamilia Orthocladiinae}

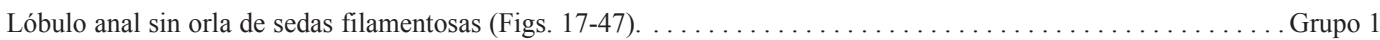

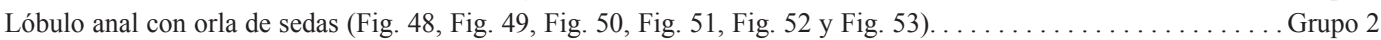

\section{GRUPO 1: Lóbulo anal sin orla de sedas.}

A. -Lóbulo anal con 2-3 macrosedas de longitud igual o como mínimo la mitad de la longitud de los lóbulos

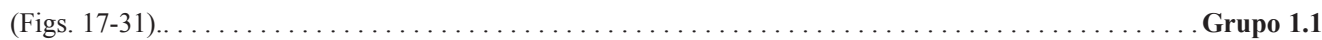

B. -Lóbulo anal sin macrosedas. Si están presentes, son delgadas y cortas o bien en forma de espinas siempre más cortas que la mitad de la longitud de los lóbulos anales

(Fig. 32, Fig. 33, Fig. 34, Fig. 35, Fig. 36, Fig. 37, Fig. 38).

Grupo 1.2

\section{Grupo 1.1 Con macrosedas.}

1. -Con un grupo de ganchos recurvados hacia adelante en la conjuntiva dorsal entre los segmentos III-IV o II-IV. Dos o

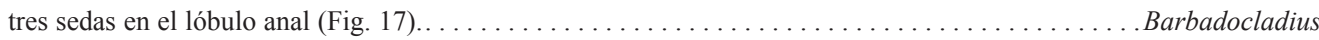

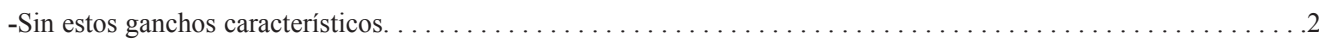

2. -Parte posterior de los tergitos con unas espinas tipo aguja muy largas, sobrepasan las conjuntivas

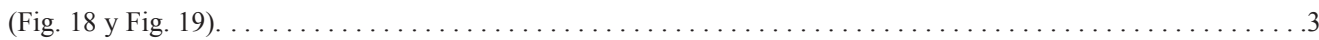

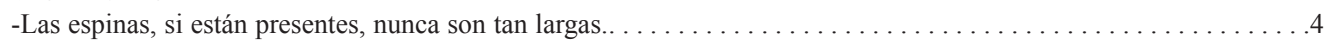

3. -Sedas frontales ausentes. Cutícula torácica granulosa. Tergito I con hilera anterior y posterior de espinas. Tergitos

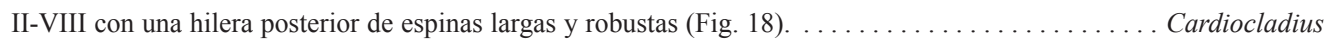
-Sedas frontales presentes. Cutícula torácica lisa. Tergito I desnudo. Tergitos II-VIII con una hilera posterior de espinas

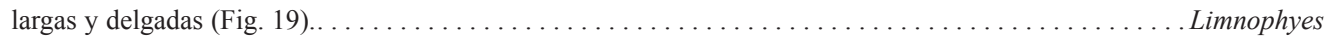

4. -Macrosedas anales en posición subapical. Lóbulo anal termina en punta (Fig. 20). . . . . . . . . . . . Parakiefferiella -Lóbulo anal diferente, por lo menos una de las macrosedas en posición apical del lóbulo anal. . . . . . . . . . . .5

5. -Fila de ganchos en el segmento II ausente. Parte posterior de los segmentos II-VIII (VII) con unas espinas muy anchas en su base o bien tubérculos que se prolongan hasta el siguiente segmento (Fig. 21, Fig. 22, Fig. 23 y Fig. 24). ... .6 -Fila de ganchos en segmento II presente, compuesta por dos hileras como mínimo. Ornamentación de los tergitos distinta, ausencia de espinas grandes en el borde posterior de los tergitos. $\ldots \ldots \ldots \ldots \ldots \ldots \ldots \ldots \ldots \ldots$

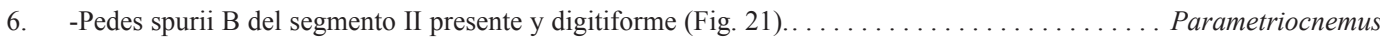

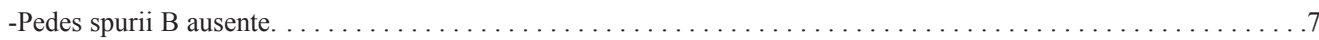

7. -Lóbulos anales en forma triangular, una de las macrosedas se sitúa en posición terminal en el lóbulo y las otras dos

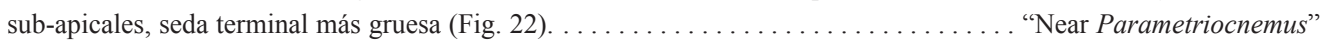


-Lóbulos anales alargados, en forma cilíndrica..

8. -Lóbulos anales paralelos. Espinas de los segmentos posteriores de menor tamaño que las de los anteriores

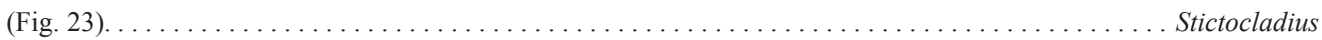

-Lóbulos anales divergentes. Espinas de los segmentos posteriores de mayor tamaño que las de los anteriores. Las del segmento VII en forma de media luna con la parte convexa situada hacia la parte anterior del segmento

(Fig. 24) . . . . . . . . . . . . . . . . . . . . . . . . . . . . . . . . . . . . Lopescladius

9. -Fila de ganchos de tamaño pequeño y ocupando la mitad del segmento, tan ancha como el campo de espinas medial del Segmento II y mucho más estrecha que el campo posterior (Fig. 25). OR más corto que las sedas precorneales,

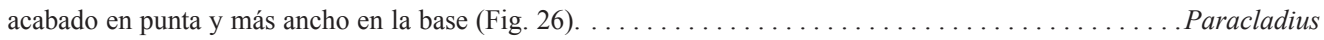
-Fila de ganchos ocupando más de la mitad del segmento, más ancho que el campo medial de espinas del segmento abdominal II (si está presente) y la mitad o menos de la anchura del campo posterior (Fig. 28). Si la fila de ganchos del segmento II es estrecha, en este caso los ganchos son de tamaño grande (Fig. 29). OR de forma y tamaño variable.

Cricotopus (en parte)

(Ver clave de morfotipos de este género a continuación).

\section{Clave para la determinación de los diferentes morfotipos de Cricotopus sp.}

1. -Con campos de espinas presentes en el segmento II (además de la fila de ganchos). PB posteriores en los segmentos

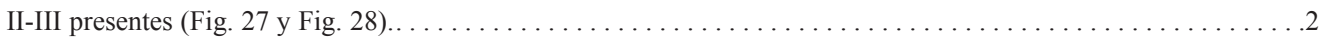

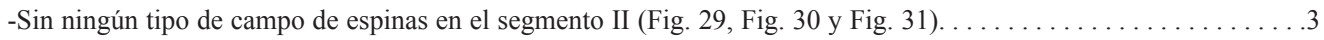

2. -Campo de espinas del segmento II reducido a un campo anterior de espinas dispersas. Sedas anales características,

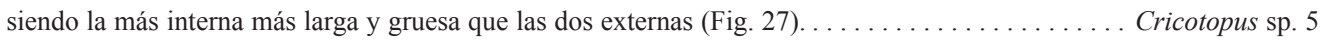
-Campos de espinas del segmento II formados por un campo medio más estrecho que la fila de ganchos y un campo posterior mucha más ancho que la fila de ganchos. Sedas anales del mismo grosor y longitud

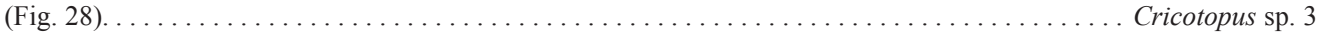

3. -Fila de ganchos del segmento II muy estrecha, ganchos muy fuertes, OR con la base más ancha y su superficie llena

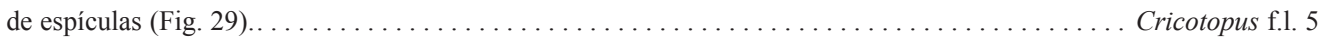
-Fila de ganchos no tan estrecha, habitualmente sobrepasando la mitad de la anchura del segmento. OR diferente. . .4

4. -OR muy pequeño de forma oval, casi imperceptible. Sedas anales más cortas que los lóbulos

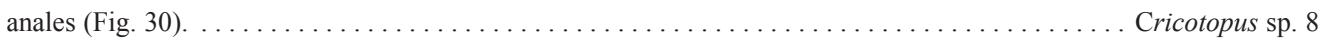
-OR variable, habitualmente de forma alargada y con espículas, pero también puede ser liso. Sedas anales de la misma

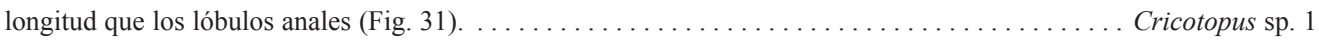

\section{Grupo 1.2: Sin Macrosedas.}

1. -Lóbulo anal con sedas cortas (inferiores a la mitad de la longitud del lóbulo anal) y robustas que parecen espinas (Fig.

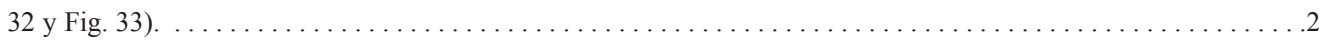
-Lóbulo anal sin sedas o si lo existen nunca son robustas. Si tiene alguna, éstas son cortas, delgadas y nunca en forma

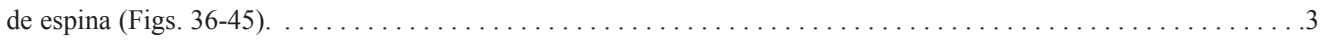

2. -Lóbulo anal con tres espinas, dos situadas en la parte distal y una en la parte media. Los segmentos II a V tienen una

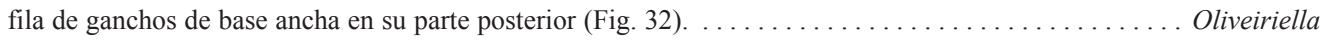
-Lóbulo anal con dos sedas en forma de espina en la parte terminal acompañadas de algunas rugosidades y una seda filiforme en la parte basal del lóbulo. Los tergitos abdominales sin ganchos de base ancha en la parte posterior; segmento II con una fila de ganchos doble, segmentos III-V con un campo central de espinas en forma de lenteja que puede conectarse por la parte central con el campo posterior que es más ancho. Campo de pequeños ganchos presente en la parte apical en los segmentos III-V (Ornamentación similar a Cricotopus).

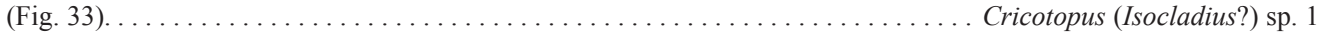

3. -Ornamentación de los tergitos con dos filas de ganchos dirigidos anteriormente en las conjuntivas de los segmentos II-V, II-IV o III-IV Lóbulos anales reducidos de tamaño similar a los sacos genitales. Pedes spurii A presentes (Figs. 38-47). Género 1

(Ver clave de morfotipos de este género al final de este grupo)

-Sin este tipo de ornamentación en los tergitos abdominales. Lóbulos anales muy reducidos. OR puede estar ausente.

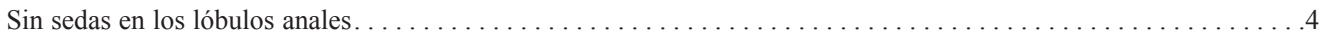


4. -Ornamentación de los tergitos con un campo de espinas posteriores muy gruesas en los tergitos IV-VIII, siendo el del

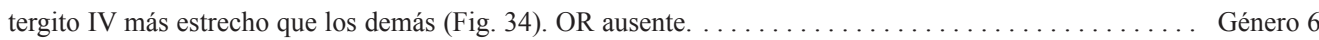

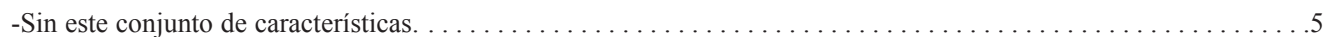

5. -Ornamentación de los tergitos abdominales restringida a unas pocas filas posteriores de espinas cortas, nunca cubiertos de espínulas de forma masiva. Pupas que viven sobre efemerópteros de la familia

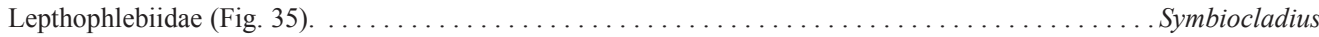

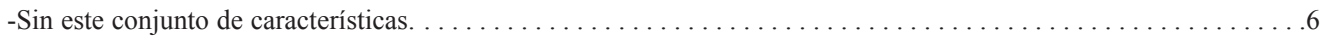

6. -Espinas intersegmentales presentes. Parte dorsal y ventral de los Tergitos abdominales con ornamentación de espínu-

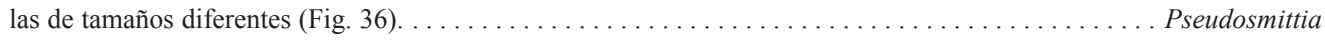
-Espinas intersegmentales ausentes. Tergitos abdominales II-VIII con una ornamentación masiva y uniforme de peque-

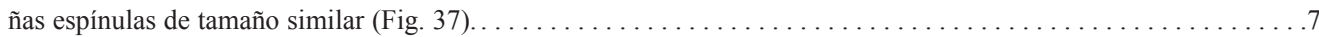

7. -Con una sola seda precorneal. Lóbulos anales alargados y rectangulares (Fig. 37)...........Gymnometriocnemus -Con dos o tres sedas precorneales. Lóbulos anales de forma trapezoidal. . . . . . . . . . . . . Bryophaenocladius

\section{Clave para la determinación de los diferentes morfotipos del Género 1 R\&C}

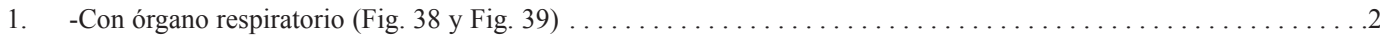

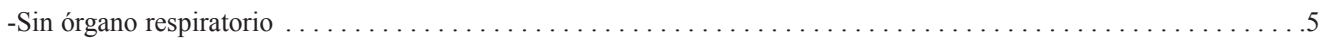

2. -OR muy pequeño y liso. Fila de ganchos y campos de espinas de los tergitos abdominales muy fuertes y oscuros. Bordes latero-anteriores de los tergitos con una marca muy oscura (Fig. 40 y Fig. 41) . . . . . . . . . . . ...3 -OR mucho más largo. Fila de ganchos y campos de espinas de los tergitos nunca tan grandes ni oscuros.......4

3. -Campo de espinas del tergito II presente. Segmentos abdominales II-VI con dos campos de espinas en la región media unidos por dos o tres espinas en la parte central. Filas de ganchos ocupando cerca de la mitad del segmento. Borde del

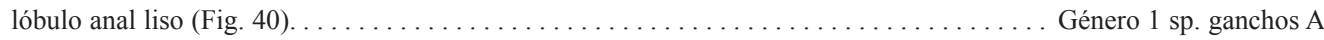
-Campo de espinas del tergito II ausente. Segmentos abdominales II-VI con un único campo de espinas en la región media. Fila doble de ganchos ocupando menos de un tercio del segmento. Borde del lóbulo

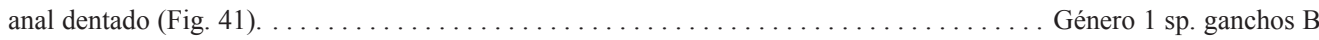

4. -OR redondeado al final y a menudo con diversos pliegues en su superficie (Fig. 38). Sin campo de espinas medio en

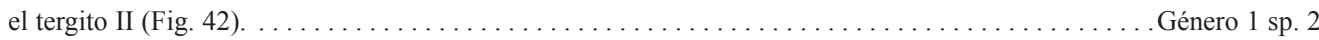
-OR más largo y terminado en punta (Fig. 39). Con un par de campos de espinas en

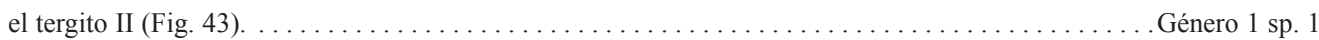

5. -Ganchos solo en los tergitos III-IV. La ornamentación de los tergitos II-VI consiste en un campo medio en forma de

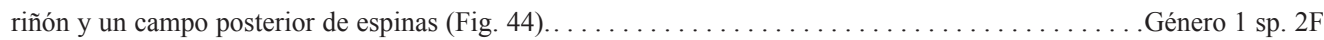

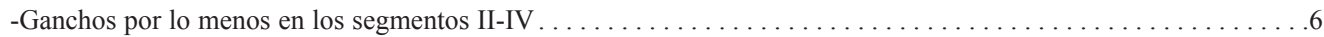

6. -Ornamentación de los tergitos III-VI con un campo de espinas medio redondeado, más estrecho que la mitad de la anchura del segmento. Fila de ganchos de los segmentos II-V estrecha como mucho ocupando la mitad de la anchura

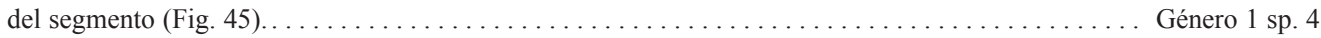

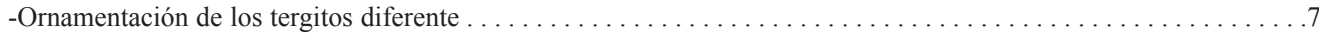

7. -Tergitos III-VI con un campo anterior de espinas que ocupa por lo menos los $2 / 3$ de la anchura del segmento. Los ganchos de los tergitos II-V ocupan más de la mitad de la anchura del mismo (Fig. 46). . . . . . . . Género 1 sp. a -Tergitos con un campo de espinas anterior y otro posterior que se juntan en su parte media en los tergitos III-VI y

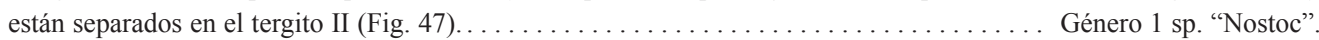

\section{GRUPO 2: Lóbulo anal con orla de sedas.}

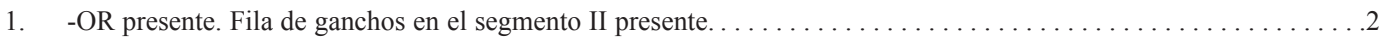

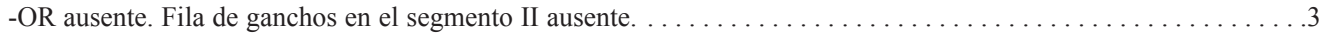

2. -Dos sedas precorneales mucho más largas que la tercera, ubicadas en un tubérculo común o en tubérculos aparte (Fig.

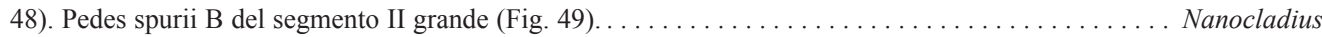
-Las tres sedas precorneales de largo similar, si dos sedas son más largas que la tercera, nunca se ubican sobre tubérculos. Pedes spurii B del segmento II ausentes o si están presentes pequeños y

redondeados (Fig. 50).

Rheocricotopus

3. -Con una sola macroseda en el lóbulo anal, la orla de sedas restringida al tercio apical, algunas sedas laterales en forma

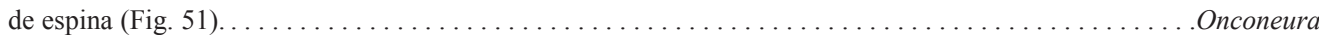


-Con tres macrosedas en el lóbulo anal, parte media del lóbulo anal sin espinas, si las sedas del lóbulo anal solo cubren

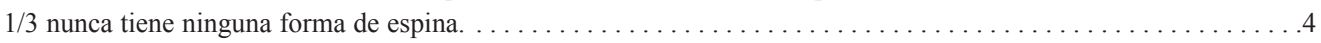

4. -Presencia de filas de perlas en el saco alar. Ornamentación del abdomen como en la Fig. 52.. . . . . . Corynoneura -Sin filas de perlas en el saco alar. Ornamentación del abdomen como en la Fig. 53 . . . . . . . . . Thienemaniella

\section{Subfamilia chironominae}

1. -OR simple, desnudo o con espinas, base en forma de domo. Tergitos abdominales (II) III-(V) VI usualmente con parches pareados o no pareados de puntos o espinas. Envoltura del ala ocasionalmente con fila de perlas (TRIBU

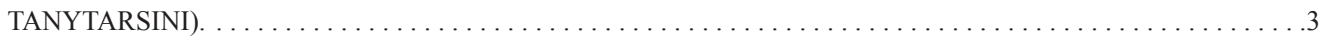
-OR ramificado, con más de dos ramas, usualmente plumoso, base en forma de anillo. Tergitos abdominales con arreglo variable de puntos o espínulas, rara vez con parches pareados. Envoltura del ala nunca con fila de perlas. . . . 2

2. -OR con muchas ramas delgadas, nunca con una rama gruesa y simple. Esternito I sin tubérculos. (TRIBU

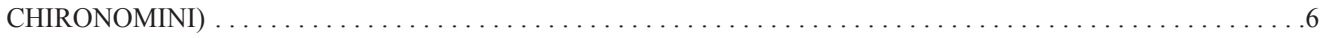
-OR con muchas ramas delgadas, además de una rama gruesa y simple. Esternito I usualmente con tubérculos antero-

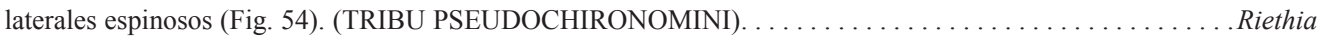

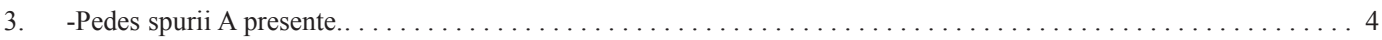

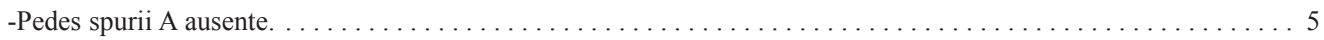

4. -Pedes spurii B ausente. Tergitos sin ornamentación de distribución uniforme. Segmentos II-V (VI) con parches

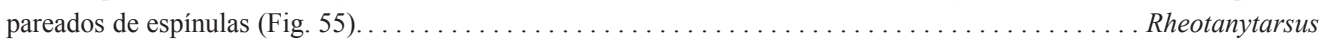
-Pedes spurii B presente. Tergitos con ornamentación de distribución uniforme (Fig. 56) . .......... Stempellinella

5. -Fila de perlas presente. Tergito IV con un parche de espinas anteromedial además de las filas de espinas laterales

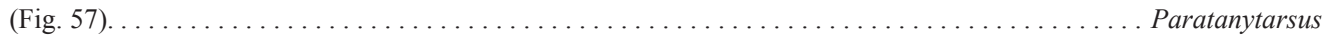
-Fila de perlas ausente. Tergitos II-VI con combinaciones variables de pares de filas longitudinales o parches de espinas

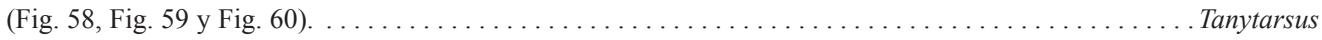

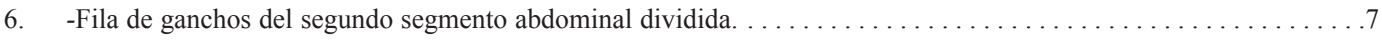

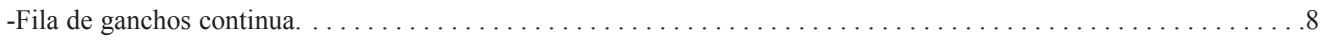

7. -Sedas frontales ausentes. OR ramificado, con una rama gruesa espinosa (Fig. 61). Pedes spurii A y B ausente. Peine

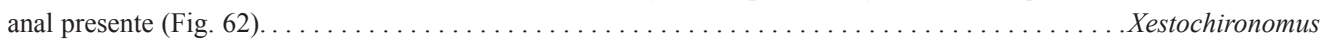
-Sedas frontales cortas. OR plumoso con numerosas ramas delgadas. Pedes spurii A presente sobre IV, pedes spurii B

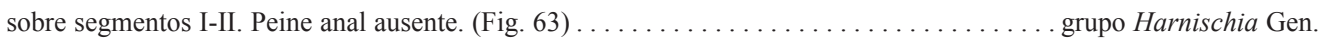

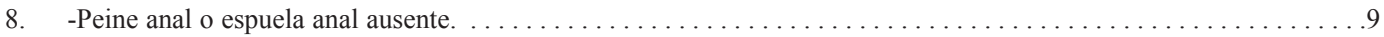
Peine anal o espuela anal presente.. . . . . . . . . . . . . . . . . . . . . . . . . . . . . . . 10

9. -Fila de ganchos sobre un lóbulo y ocupando la $1 / 2$ del segmento. Pedes spurii A y B presentes. Segmento VIII con 4

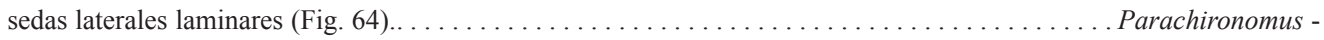
-Fila de ganchos ocupando menos de la $1 / 2$ del segmento. Pedes spurii B ausente. Segmento VIII con 5 sedas laterales

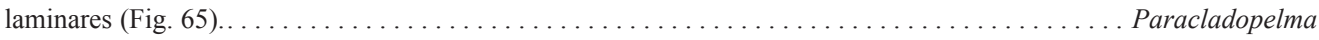

10. -Ornamentación de los tergitos II-VI en forma de una mancha extensiva de espinas. Tubérculos cefálicos cónicos. OR plumoso, anillo basal notable y bilobulado. Espuela anal compuesta por muchos dientes (Fig. 66).. .... . Chironomus -Ornamentación de los segmentos II-VI nunca de forma extensiva, en el caso de que algún segmento (III) lo esté, las

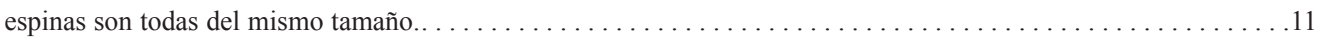

11. -Esternitos I-II o I-III con unas filas transversales de espinas incoloras dirigidas posteriormente (Fig. 67). Peine anal

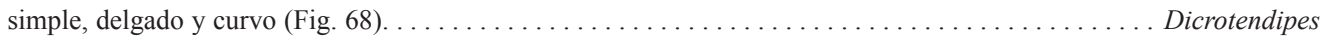

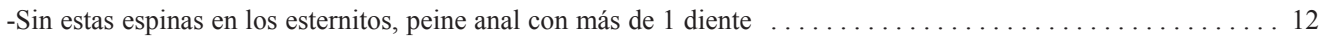

12. -Con 3 sedas laminares en el segmento V. OR muy ramificado, pero no plumoso (Fig. 69). Peine anal compuesto por

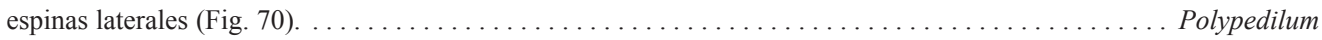
-Con 4 sedas laminares en el segmento V. OR poco ramificado. Peine anal compuesto por 4 dientes (Fig. 71). 

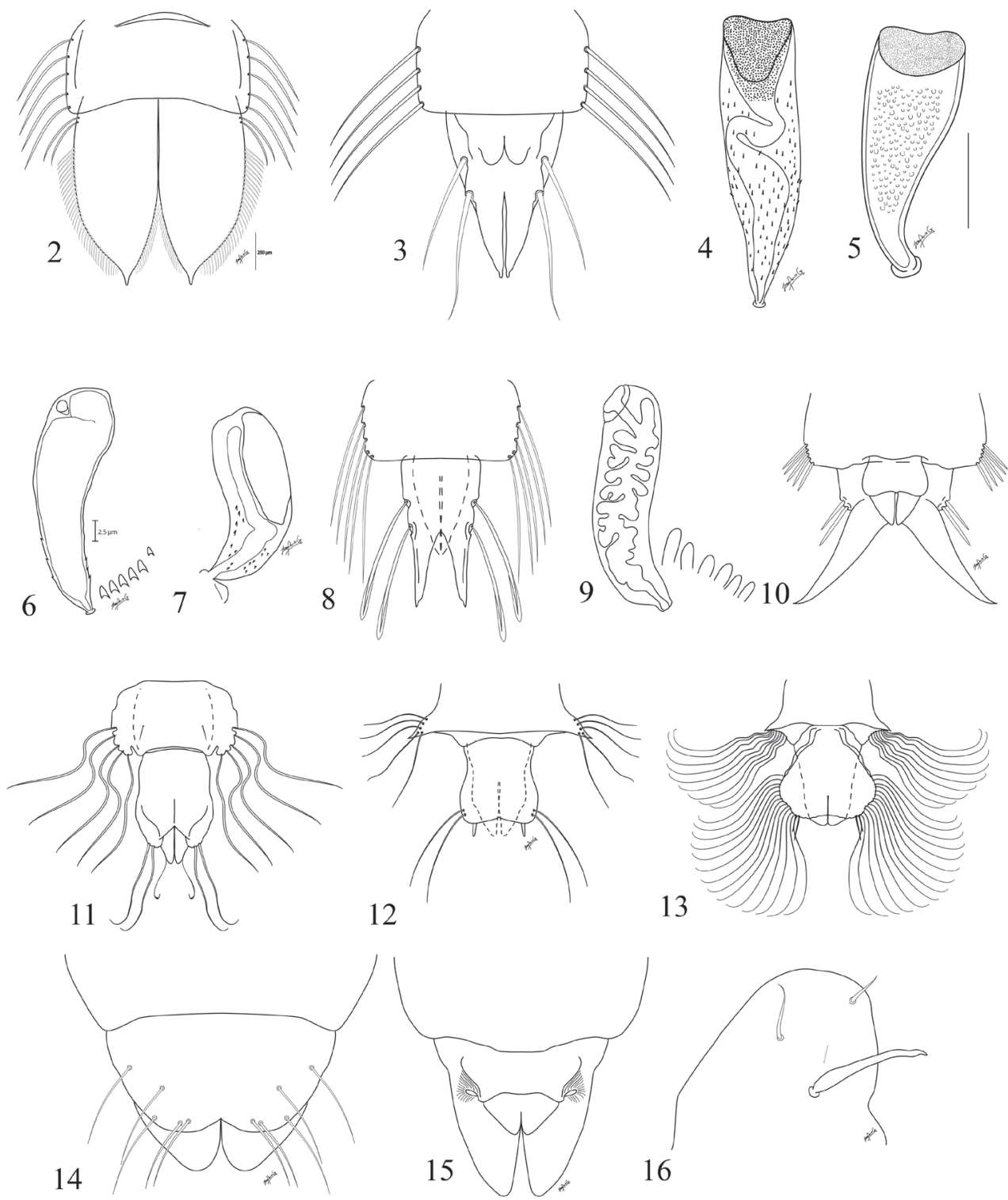

16

Figs. 2-16. Tanypodinae: 2. Segmento abdominal VIII y lóbulos anales de Alotanypus. 3. Ídem de Pentaneura. 4. Órgano respiratorio (OR) de Alotanypus. 5. OR de Apsectrotanypus 6. OR de Labrundinia. 7. OR de Pentaneura. 8. Segmento abdominal VIII y lóbulos anales de Larsia. 9. OR de Hudsonimyia. 10. Segmento abdominal VIII y lóbulos anales de Hudsonimyia. Podonominae: 11. Segmento abdominal VII y lóbulos anales de Podonomopsis. 12. Ídem de Parochlus. 13. Ídem de Podonomus. Diamesinae Tribu Heptagyinii: 14. Segmento abdominal VII y lóbulos anales de Paraheptagyia. 15. Segmento abdominal VII y lóbulos anales de Limaya. 16. Parte anterior del tórax del mismo género con las dos sedas engrosadas propias del mismo.

Figs. 2-16. Tanypodinae: 2. Alotanypus anal lobe and abdominal segment VIII. 3. Ídem Pentaneura. 4. Thoracic horn (TH) of Alotanypus. 5. TH of Apsectrotanypus. 6. TH of Labrundinia. 7. TH of Pentaneura. 8. Larsia anal lobe and abdominal segment VIII. 9. TH of Hudsonimyia. 10. Hudsonimyia anal lobe and abdominal segment VIII. Podonominae: 11. Podonomopsis anal lobe and posterior abdominal segments. 12. Ídem Parochlus. 13. Ídem Podonomus. Diamesinae Tribu Heptagyinii: 14. Paraheptagyia anal lobe and posterior abdominal segments. 15. Ídem Limaya. 16. Anterior area of Limaya thorax. 

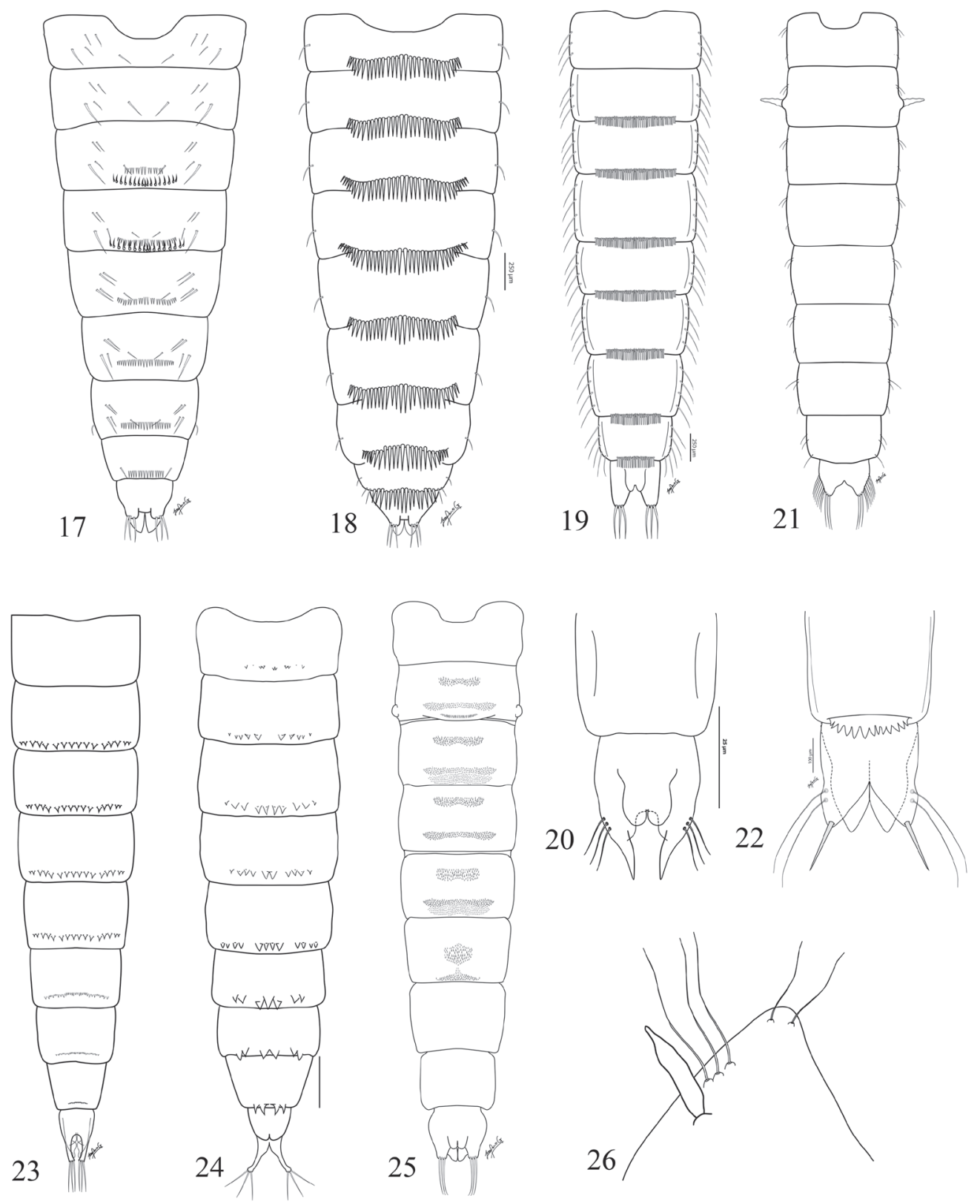

Figs. 17-26. Orthocladiinae: 17. Ornamentación del abdomen del género Barbadocladius. 18. Ídem de Cardiocladius . 19. Ídem de Lymnophyes. 20. Segmento abdominal VIII y lóbulos anales de Parakiefferiella. 21. Ornamentación del abdomen de Parametriocnemus. 22. Segmento abdominal VIII y lóbulos anales de "Near Parametriocnemus" 23. Ornamentación del abdomen de Stictocladius. 24. Ídem de Lopescladius. 25. Ídem de Paracladius. 26. Parte anterior del tórax del mismo género.

Figs. 17-26. Orthocladiinae: 17. Abdominal segments and anal lobe of Barbadocladius. 18. Ídem Cardiocladius. 19. Ídem Lymnophyes. 20. Abdominal segment VIII and anal lobe of Parakieferiella. 21. Abdominal segments and anal lobe of Parametriocnemus. 22. Abdominal segment VIII and anal lobe of genus "Near Parametriocnemus". 23. Abdominal segments and anal lobe of Stictocladius. 24. Ídem Lopescladius. 25. Ídem Paracladius. 26. Anterior area of Paracladius thorax. 

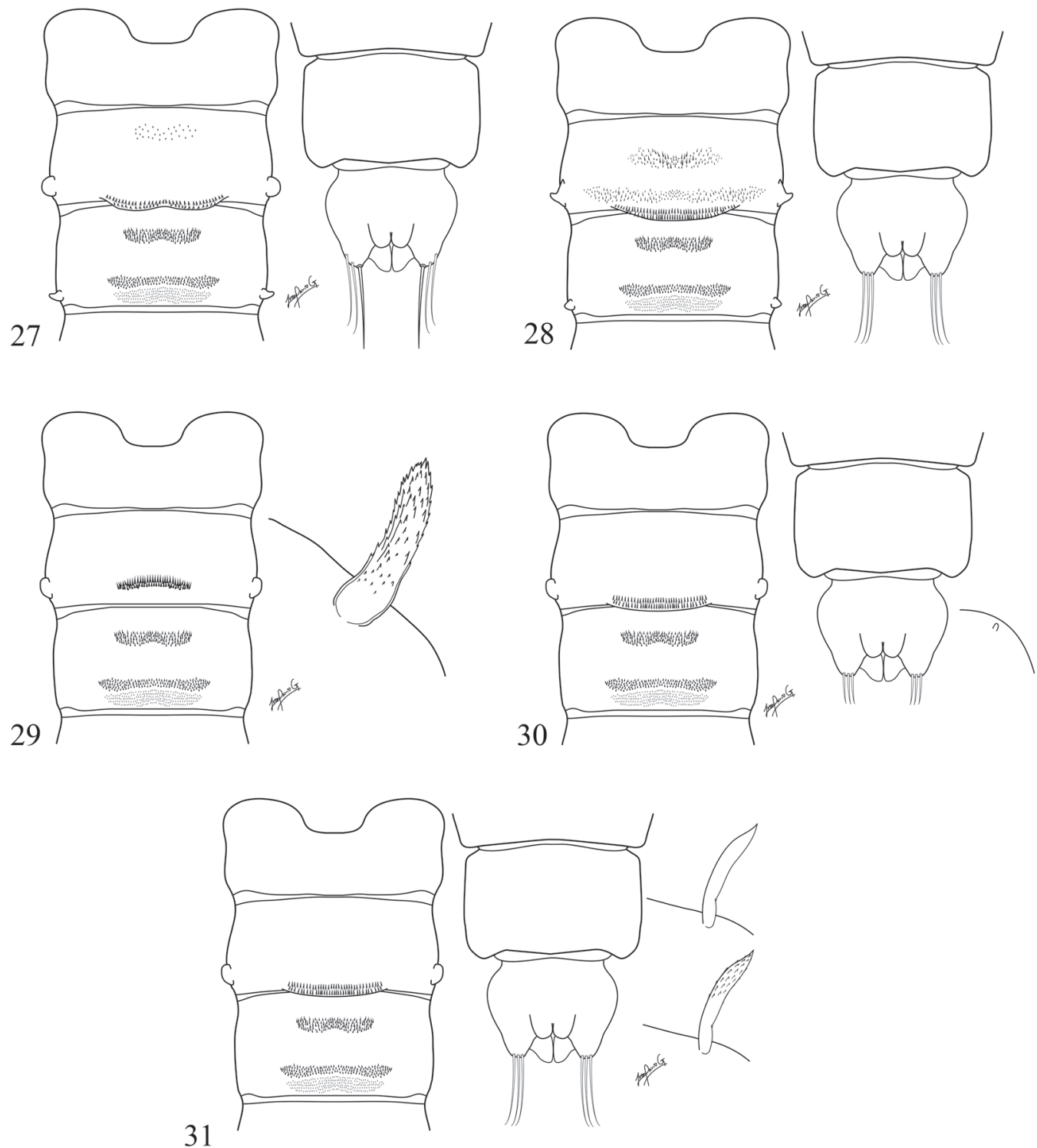

Figs. 27-31. Orthocladiinae: Género Cricotopus: 27. Segmentos abdominales I-III y parte terminal de Cricotopus sp. 5. 28. Ídem de Cricotopus sp. 3. 29. Segmentos abdominales I-III y OR de Cricotopus f.1. 5. 30. Segmentos abdominales I-III y parte terminal de Cricotopus sp. 8. 31. Segmentos abdominales I-III, parte terminal de la pupa y dos formas de OR de Cricotopus sp.1.

Figs. 27-31. Orthocladiinae: Genus Cricotopus: 27. Abdominal segments I-III \& VIII and anal lobe of Cricotopus sp.5. 28. Ídem Cricotopus sp.3. 29. Abdominal segments I-II and TH of Cricotopus f.1. 5. 30. Abdominal segments I-III \& VIII and anal lobe of Cricotopus sp. 8. 31. Abdominal segments I-III \& VIII, anal lobe and TH of Cricotopus sp. 1. 

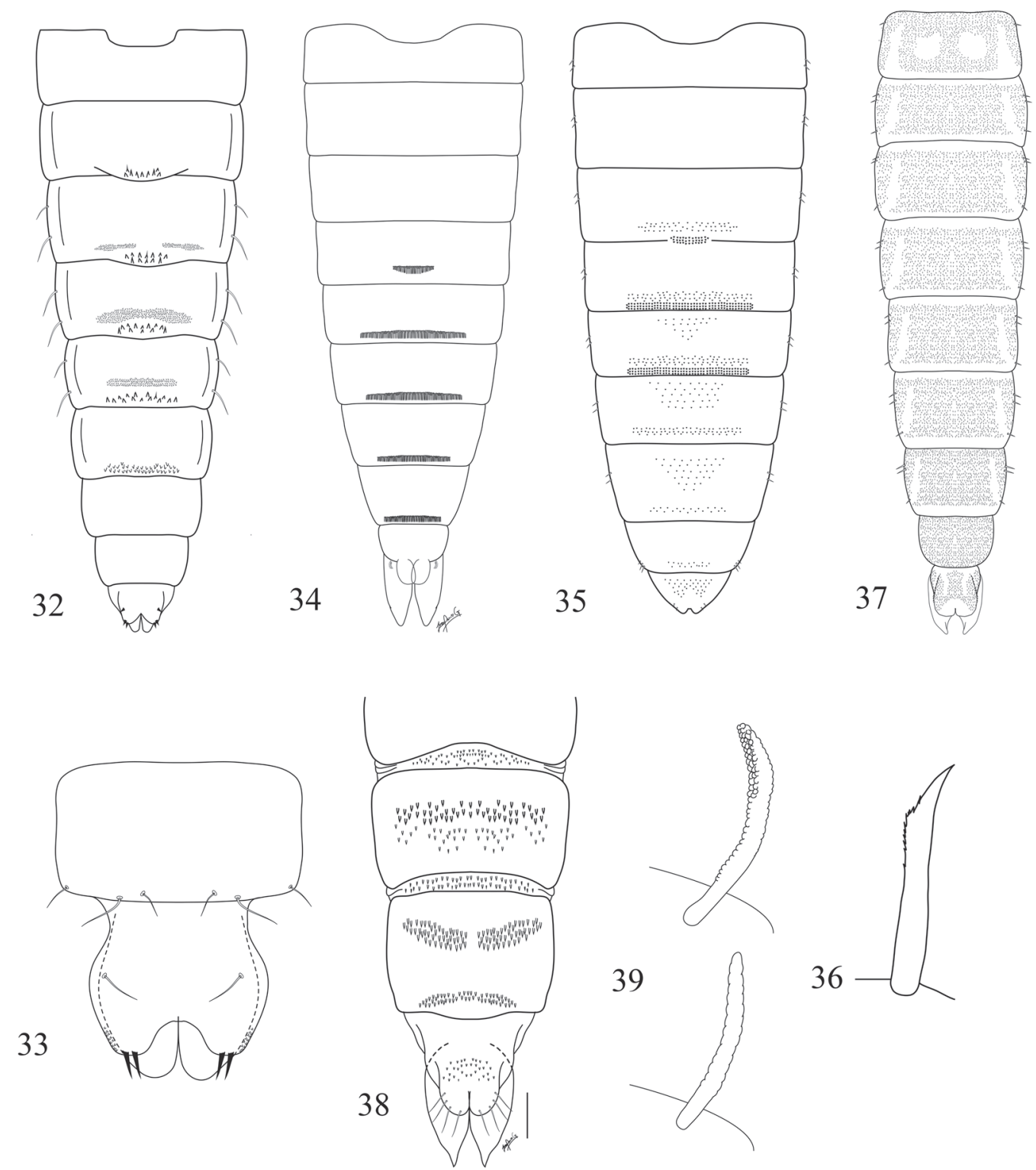

Figs. 32-39. Orthocladiinae: 32. Ornamentación del abdomen del género Oliveiriella. 33. Segmento abdominal VIII y lóbulos anales de Cricotopus (Isocladius?) sp. 1. 34. Ornamentación del abdomen del Género 6. 35. Ídem Symbiocladius. 36. Segmentos abdominales VII - VIII y lóbulos anales de Pseudosmittia. 37. Ornamentación del abdomen de Gymnometriocnemus. 38. OR del Género 1 sp. 2. 39. OR del Género 1 sp. 1.

Figs. 32-39. Orthocladiinae: 32. Abdominal segments and anal lobe of Oliveiriella. 33. Anal lobe and abdominal segment VIII of Cricotopus (Isocladius?) sp. 1.34. Abdominal segments and anal lobe of genus 6. 35. Ídem Symbiocladius. 36. Anal lobe and abdominal segments VII - VIII of Pseudosmittia. 37. Abdominal segments and anal lobe of Gymnometriocnemus. 38. TH of genus 1 sp. 2. 39. TH of Genus 1 sp. 1. 

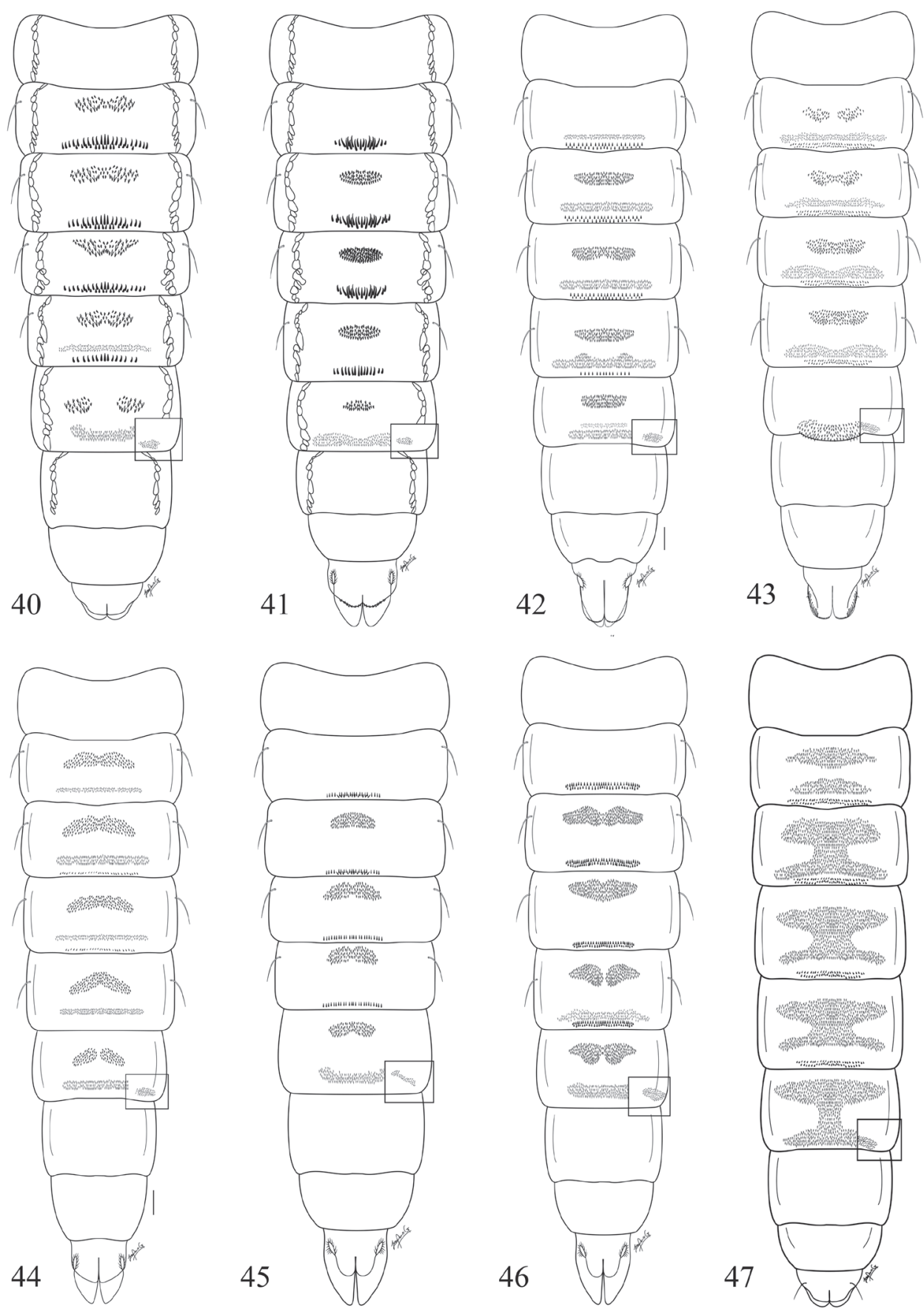

Figs. 40-47. Orthocladiinae: Género 1: 40. Ornamentación del abdomen del Género $1 \mathrm{sp}$. ganchos A. 41. Ídem de Género 1 sp. ganchos B. 42. Ídem de Género 1 sp. 2. 43. Ídem de Género 1 sp. 1. 44. Ídem de Género 1 sp. 2F. 45. Ídem de Género 1 sp. 4. 46. Ídem de Género 1 sp. a. 47. Ídem de Género 1 sp. "Nostoc".

Figs. 40-47. Orthocladiinae: Genus 1: 40. Abdominal segments of Genus 1 sp. "ganchos A". 41. Ídem Genus 1 sp. "ganchos B". 42. Ídem Genus 1 sp.2. 43. Ídem Genus 1 sp. 1. 44. Ídem Genus 1 sp. 2F. 45. Ídem Genus 1 sp. 4. 46. Ídem Genus 1 sp. a. 47. Ídem Genus 1 sp. "Nostoc". 


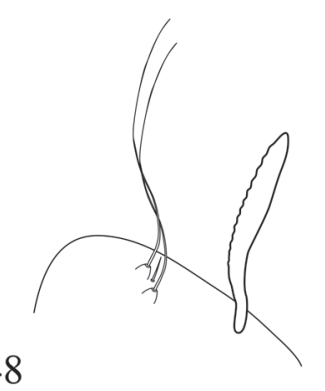

48

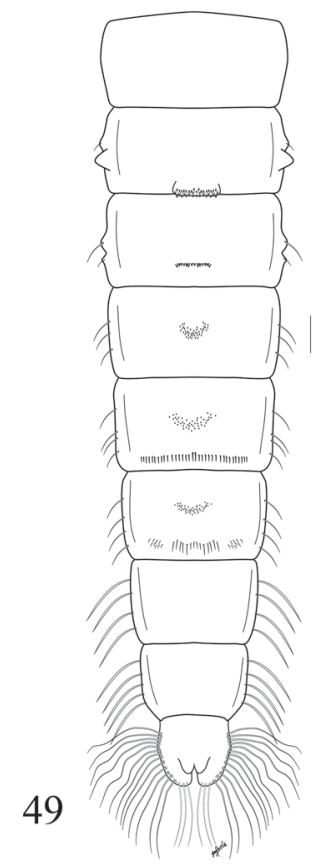

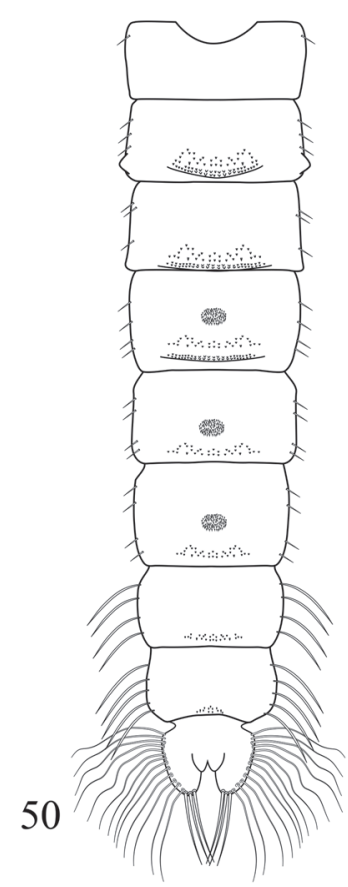
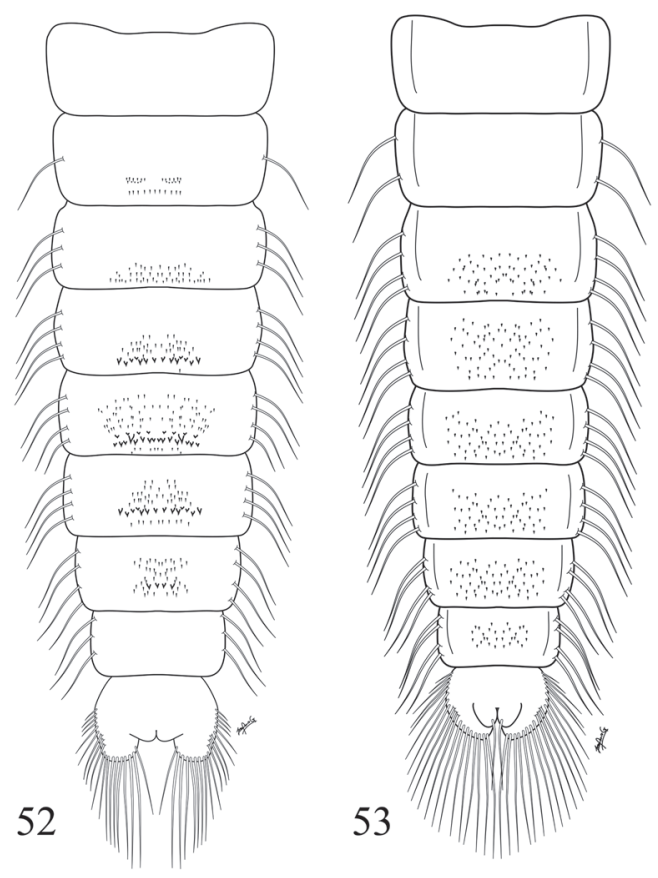

Figs. 48-53. Orthocladiinae: 48. Parte anterior del tórax del género Nanocladius. 49. Ornamentación del abdomen del mismo género. 50. Ídem de Rheocricotopus. 51. Segmento abdominal VIII y lóbulos anales de Onconeura. 52. Ornamentación del abdomen de Corynoneura. 53. Ídem de Thienemanniella.

Figs. 48-53. Orthocladiinae: 48. Anterior area of Nanocladius thorax. 49. Abdominal segments of Nanocladius. 50. Ídem Rheocricotopus. 51. Anal lobe and abdominal segment VIII of Onconeura. 52. Abdominal segments of Corynoneura. 53. Ídem Thienemanniella. 

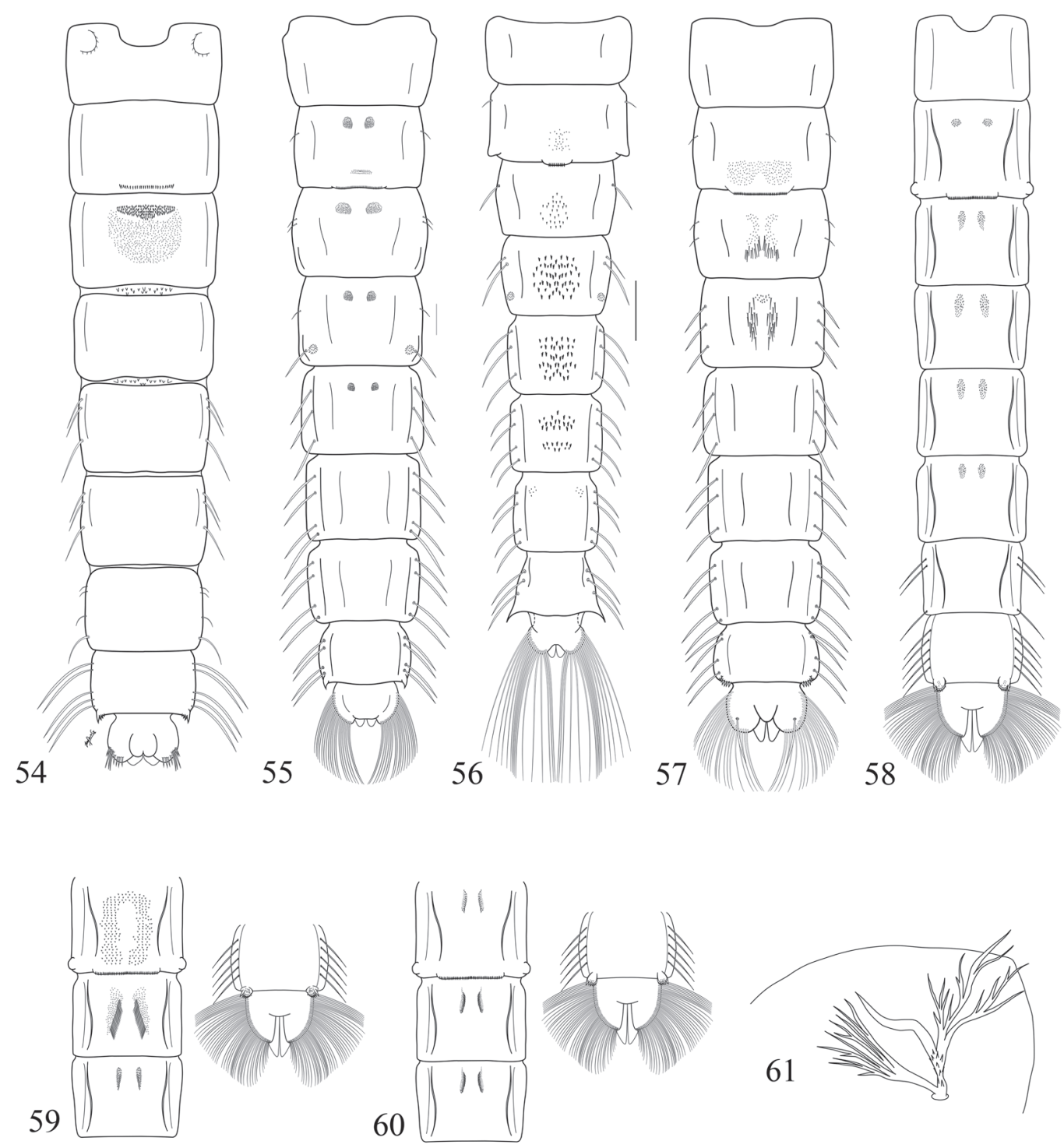

Figs. 54-61. Chironominae: 54. Ornamentación del abdomen de Riethia. 55. Ídem de Rheotanytarsuss. 56. Ídem del género Stempellinella. 57. Ídem del género Paratanytarsus. 58-60 Tanytarsus: 58. Ornamentación del abdomen. 59-60. Segmentos II a IV y parte terminal del abdomen de dos especies diferentes de Tanytarsus. 61. OR de Xestochironomus.

Figs. 54-61. Chironominae: 54. Abdominal segments of Riethia. 55. Ídem Rheotanytarsus. 56. Ídem Stempellinella. 57. Ídem Paratanytarsus. 58-60 Tanytarsus: 58. Abdominal segments. 59-60. Anal lobe and abdominal segments II-IV of two species of Tanytarsus. 61. TH of Xestochironomus. 

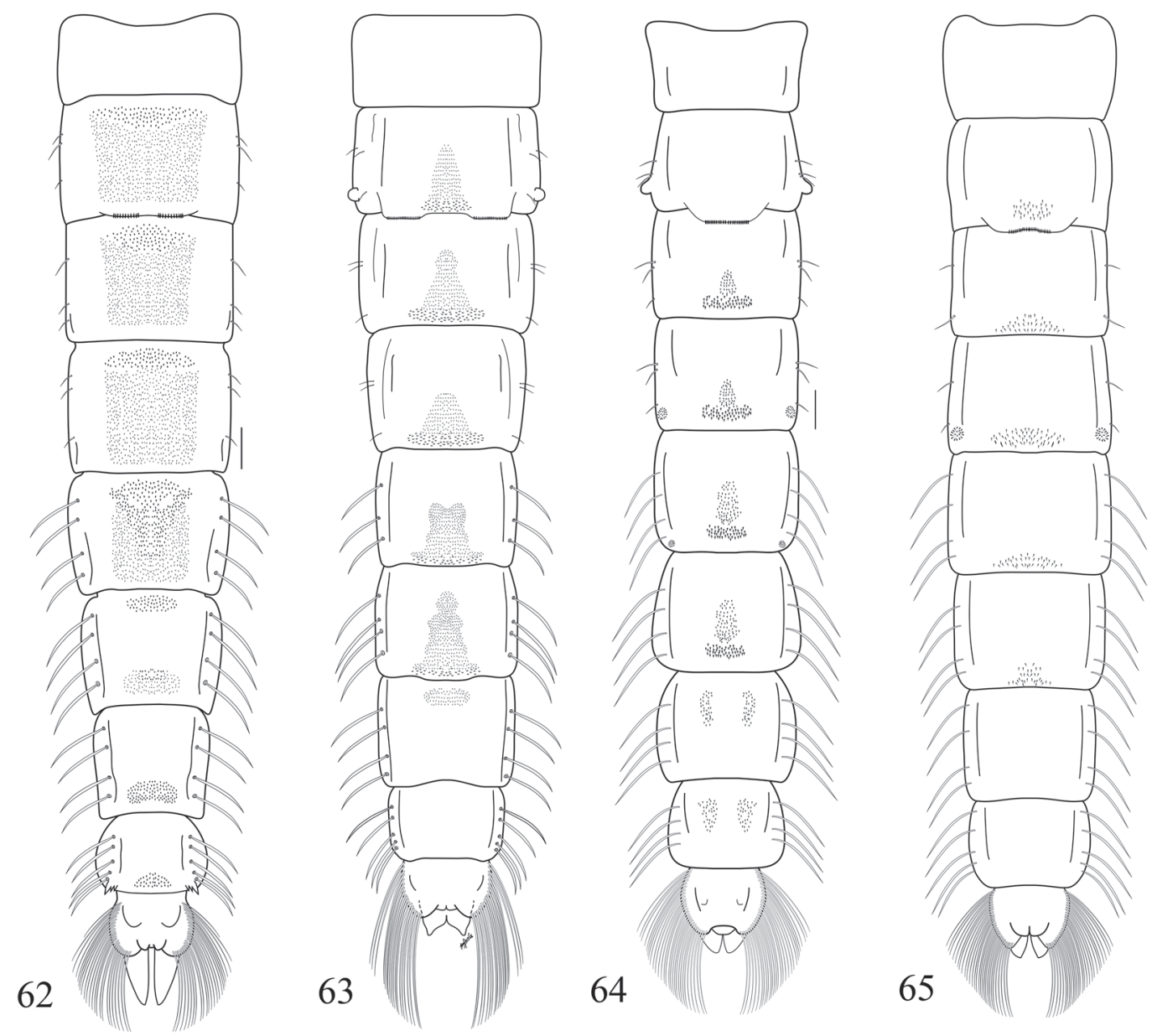

Figs. 62-65. Chironominae: 62. Ornamentación del abdomen de Xestochironomus. 63. Ídem de Harnischia. 64. Ídem de Parachironomus 65. Ídem de Paracladopelma.

Figs. 62-65. Chironominae: 62. Abdominal segments of Xestochironomus. 63. Ídem Hanischia. 64. Ídem Parachironomus. 65. Ídem Paracladopelma. 

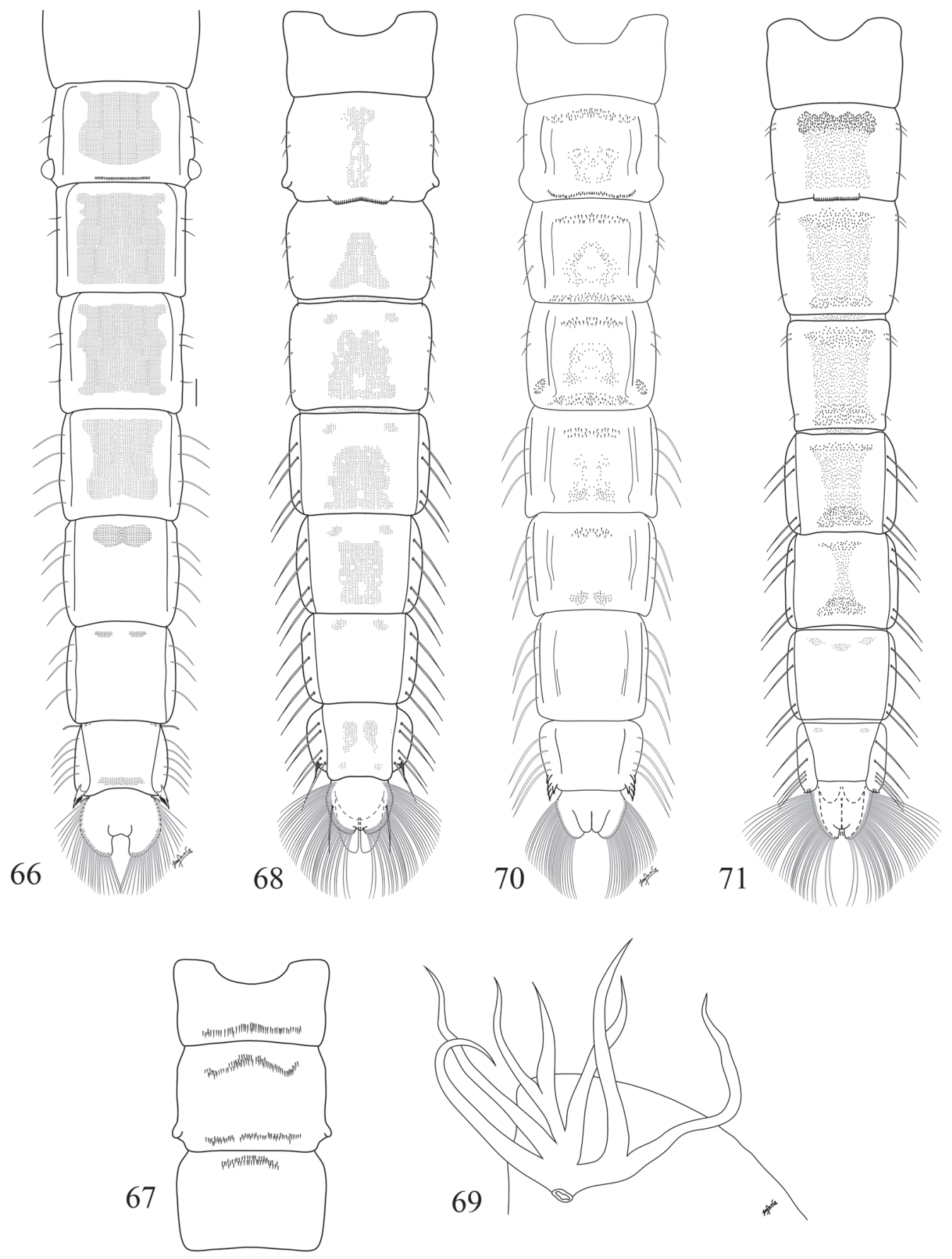

Figs. 66-71. Chironominae: 66. Ornamentación del abdomen de Chironomus. 67. Esternitos I-III de Dicrotendipes. 68. Ornamentación del abdomen del mismo género. 69. OR de Polypedilum. 70. Ornamentación del abdomen del mismo género. 71. Ídem del género Stenochironomus.

Figs. 66-71. Chironominae: 66. Abdominal segments of Chironomus. 67. Sternites I-III of Dicrotendipes. 68. Abdominal segments of Dicrotendipes. 69. TH of Polypedilum. 70. Abdominal segments of Polypedilum. 71. Ídem Stenochironomus. 


\section{DISCUSIÓN}

En las recolecciones de muestras bentónicas de macroinvertebrados, además de las larvas de quironómidos se encuentran a veces sus pupas o las exuvias que quedan flotando, una vez que el adulto abandona el agua. La recolección de exuvias mediante redes de deriva posibilita una mejor identificación de los géneros y especies de quironómidos que viven en el ecosistema estudiado. Hay que tener en cuenta que el muestreo y la elaboración de micropreparados de exuvias de quironómidos es más rápido que el de sus larvas y proporciona una mayor precisión en la determinación de géneros y especies, tal como ha sido puesto de manifiesto en otras zonas geográficas cuya fauna era también desconocida, por ejemplo en Australia (Cranston, 2000). Así mismo posibilita el uso de las exuvias para estudios ecológicos y planes de monitoreo y de control de calidad de los sistemas fluviales. De esta manera, este trabajo enriquece el conocimiento del grupo en la zona altoandina tropical y facilita su uso en estudios taxonómicos y ecológicos. Esta clave se centra en las especies encontradas en ambientes lóticos, llegando hasta el nivel de género (con descripción de algunas morfoespecies en algunos géneros). Dado que a nivel de género los rasgos biológicos de las especies pueden llegar a diferir, entendemos que la clave puede ser muy útil para aquellos que pretendan utilizar los quironómidos en estudios de tipo funcional.

De la lista de especies encontradas, se puede destacar la relativa pobreza de géneros respecto a otros estudios realizados en ríos de altitud de otros lugares del mundo (e.g. Lencioni, Marziali \& Rossaro, 2011), así como la ausencia de muchos géneros presentes en la Patagonia (Brundin, 1966). De acuerdo con las escasa información que sobre quironómidos se dispone en la región, podemos intuir que hay una pérdida o disminución de taxones de origen Gondwánico a medida que disminuye la latitud, lo que concuerda con lo expuesto por Cranston et al. (2010). Aparentemente esto no se ve compensado por una mayor presencia de géneros Holárticos, pero es clara la dominancia de la tribu Orthocladiinae sobre el resto de géneros encontrados, por lo menos en nuestras recolecciones en ríos altoandinos. Por otra parte algunos géneros que no se encuentran en la Patagonia ni en Norteamérica al parecer se han diversificado mucho a nivel de especie en la zona, como en el caso del Género 1 (Roback \& Coffman, 1983), o los géneros con una distribución más amplia como Cricotopus o algunos de los miembros de la Tribu Corynoneurinii (Thienemaniella, Corynoneura y Onconeura). Cabe resaltar que las especies del Género 1 presentan caracteres morfológicos muy diferentes entre ellas, por lo que es probable que este grupo tan abundante de quironómidos de los ríos altoandinos pueda ser un complejo de géneros de manera similar al grupo Harnischia. Debido a esto, los estudios genéticos que estamos realizando en la actualidad, nos pueden dar muchas pistas sobre si realmente son especies de un mismo género o hay diferentes géneros (Prat et al. 2013). Por ello ahora mismo es difícil saber si esta zona representa, al igual que para las plantas o los animales, una zona de gran diversificación y de alta biodiversidad o no para los quironómidos.

\section{AGRADECIMIENTOS}

Este trabajo es dedicado a la memoria del profesor E. J. Fittkau y no hubiera sido posible sin la financiación que el Ministerio de Educación y Cultura y Deporte ha proporcionado a N. Prat mediante los proyectos CGL2005-24168-E; CGL 2006-04333 y CGL2012-16635 y la financiación de la Agencia Española de Cooperación Internacional, proyectos: D/011294/07; D/025074/09. También a dos revisores anónimos y a los editores de la revista por sus comentarios y sugerencias.

\section{RESUMEN}

Chironomidae es una familia cosmopolita de dípteros nematóceros que comprende más de 20000 especies descritas. Sin embargo, la diversidad de géneros y especies presentes en la región andina del Neotrópico es poco conocida. Por tal motivo se desarrolló una clave 
taxonómica para identificar las pupas y exuvias pupales de 46 géneros de la familia Chironomidae presentes en ríos de alta montaña ubicados en la cordillera de los Andes por encima de los $2000 \mathrm{msnm}$. Se utilizó material de colección obtenido de levantamientos faunísticos y estudios de biomonitoreo en diferentes ríos y quebradas de Colombia, Ecuador y Perú. La clave incluye ilustraciones y breves descripciones taxonómicas de todos los géneros incluyendo algunos que aún no han sido descritos formalmente. Para estos últimos se siguió la nomenclatura usada por Roback \& Coffman (1983). Para los géneros Cricotopus y Género 1 se incluyen claves para la determinación de las morfoespecies más comunes. En el ensamblaje de quironómidos de la región altoandina domina la subfamilia Orthocladiinae (22 géneros) seguida por Chironominae (13 géneros), patrón que contrasta con la dominancia de Chironominae en las zonas de menor altura de la región Neotropical. Se encontraron seis géneros pertenecientes a la subfamilia Tanypodinae, mientras que para Podonominae y Diamesinae estuvieron presentes tres y dos géneros respectivamente. Esta clave podría llegar a ser muy útil en estudios de la deriva y programas de biomonitoreo de los ríos altoandinos neotropicales.

Palabras clave: Chironomidae, ríos altoandinos, pupas, exuvias pupales, deriva.

\section{REFERENCIAS}

Acosta, R. (2009). Estudio de la cuenca altoandina del río Cañete (Perú). (Tesis de Doctorado). Universidad de Barcelona, España. Recuperado de www.ub.edu/ riosandes/docs/TESIS_RAUL_ACOSTA.pdf

Acosta, R. \& Prat, N. (2010). Chironomid assemblages in high altitude streams of the Andean region of Peru. Fundamental and Applied Limnology, 177(1), 57-79.

Brundin, L. (1966). Transantarctic relationships and their significance, as evidenced by Chironomid midges, with a monograph of the subfamilies Podonominae and Aphroteniinae and the austral Heptagyidae. Kungl. Svenska Vetenskapsakademiens Handlingar, 1, 1-472.

Coffman, W. P. (1986). The pupae of Chironomidae (Diptera) of the Holartic region. Key to subfamilies. In T. Wiederholm (Ed.), Chironomidae of the Holartic region: Key and diagnoses. Part 2. Pupae (pp. 9-12). Entomological Scandinavica.

Cranston, P. S. (2000). The electronic guide of Chironomidae of Australia. Retrieved from http://apes.skullisland.info/node/3

Cranston P. S., Hardy, N. B., Morse, G. E, Pusledik, L., \& McCluen, S. R. (2010). When molecules and morphology concur: the "Gondwanan" midges (Diptera: Chironomidae). Systematic Ecology, 35, 635-648.
Cranston, P. \& Krosch, M. (2011). Barbadocladius Cranston \& Krosch, a new genus of Orthocladiinae (Diptera: Chironomidae) from South America. Neotropical Entomology, 40(5), 560-567.

García, P. \& Añón-Suárez, D. (2007). Community structure and phenology of chironomids (Insecta Chironomidae) in a Patagonina Andean Stream. Limnologica, 37, 109-117.

Jacobsen, D. (2008). Tropical High-Altitude Streams. In D. Dudgeon (Ed.), Tropical stream ecology (pp. 219256). London, UK: Elsevier Science.

Krosh, M. N., Baker, A. M. P., \& Cranston, P. (2011). Systematics and biogeography of the Gondwanan Orthocladiinae (Diptera: Chironomidae). Molecular Phylogenetics and Evolution, 59(2), 458-468.

Kuhn, J., Andino, P., Calvez, R., Espinosa, R., Hamerlik, L., Vie, S., Dangles, O., \& Jacobsen, D. (2011). Spatial variability in macroinvertebrate assemblages along and among neighbouring equatorial glacier-fed streams. Freshwater Biology, 56(1), 2226-2244.

Lencioni, V., Marziali, L., \& Rossaro, B. 2011. Diversity and distribution of chironomids (Diptera, Chironomidae) in Alpine and pre-Alpine springs (Northern Italy). Journal of Limnology, 70 (Supp. 1), 106-121.

Medina, A., Scheibler, E. E., \& Paggi, A. C. (2008). Distribución de Chironomide (Diptera) en dos sistemas fluviales ritrónicos (Andino-serrano) de Argentina. Revista de la Sociedad Entomológica de Argentina, 67(1-2), 69-79.

Ospina-Torres, R., Riss, W., \& Ruiz-Moreno, J. L. (1999). Guía para la identificación genérica de larvas de quironómidos (Diptera: Chironomidae: Orthocladiinae) de la sabana de Bogotá. In G. Amat-García, G. Andrade, \& F. Fernández (Eds.), Insectos de Colombia Vol. II (pp. 363-384). Bogotá: Academia Colombiana de Ciencias Exactas, Físicas y Naturales.

Paggi, A. (2009). Diptera. Chironomidae. In E. Domínguez \& H. R. Fernández (Eds.), Macroinvertebrados bentónicos sudamericanos. Sistemática y Biología (pp. 383-409). Tucumán: Fundación Miguel Lillo.

Pinder, L. C. V. \& Reiss, F. (1986). The pupae of Chironomidae (Diptera) of the Holartic region. Introduction. In T. Wiederholm (Ed.), Chironomidae of the Holartic region: Key and diagnoses. Part 2. Pupae (pp. 5-7). Entomological Scandinavica, (Supp. 28).

Prat, N., Acosta, R., Villamarín, C., \& Rieradevall, M. (2011). Guía para el reconocimiento de las larvas de Chironomidae (Diptera) de los ríos altoandinos de Ecuador y Perú. Recuperado de: http://www.ub.edu/ riosandes/index.php/guiachiros.html

Prat, N., Ribera, C., Rieradevall, M., Villamarin, C., \& Acosta, R. (2013). Distribution, abundance and molecular analysis of Barbadocladius Cranston \& Krosch (Diptera, Chironomidae) in tropical, high altitude 
Andean streams and rivers. Neotropical Entomology, 42, 607-617.

Roback, S. S. (1970). Podonominae of Ecuador, with Notes on the Sense Organ and Pupal Respiratory Organs (Diptera, Chironomidae). Journal of the New York Entomological Society, LXXVIII, 148-169.

Roback, S. S. \& Coffman, W. P. (1983). Results of the Catherwood Bolivian-Peruvian Altiplano Expedition. Part II. Aquatic Diptera including montane Diamesinae and Orthocladiinae (Chironomidae) from Venezuela. Proceedings of Natural Sciences of Philadelphia, 135, 9-79.

Ruiz-Moreno, J. L., Ospina-Torres, R., \& Riss, W. (2000a). Guía para la identificación genérica de larvas de Quironómidos (Diptera Chironomidae) de la sabana de Bogotá. II Subfamilia Chironominae. Caldasia, 22(1), 15-33

Ruiz-Moreno, J. L., Ospina-Torres, R., Gomez-Sierra, H., \& Riss, W. (2000b). Guía para la identificación genérica de larvas de Quironómidos (Diptera Chironomidae) de la sabana de Bogotá. III subfamilias Tanypodinae, Podonominae y Diamesinae. Caldasia, 22(1), 34-60.

Saether, O. \& Cranston, P. S. (2012). Neotropical Stictocladius Edwards (Diptera: Chironomidae). Neotropical Entomology, 41, 124-149.

Spies, M. \& Reiss, F. (1996). Catalog and bibliography of Neotropical and Mexican Chrionomidae. Spixiana, (Suppl. 22), 61-119.

Tejerina, E. G. \& Molineri, C. (2007). Comuniddes de Chironomidae (Diptera) en arroyoos de montaña del NOA: comparación entre Yungas y Monte. Revista de la Sociedad Entomológica de Argentina, 66(3), 169-177.

Tejerina, E. G. \& Paggi, A. C. (2009). A new neotropical species of Oliveiriella Wiedenburg \& Fittkau (Diptera, Chironomidae) from Argentina, with description of all life stages. Aquatic Insects, 31(2), 91-98.

Tejerina, E. G. \& Malizia, A. (2012). Chironomidae (Diptera) larvae assemblages differ along an altitudinal gradient and temporal periods in a subtropical montane stream in Nortwest Argentina. Hydrobiologia, $686,41-54$.
Trivino-Strixino, S., Roque, F. O., \& Cranston, P. (2009). Redescription of Riethia trucatocaudata (Edwards, 1931) (Diptera: chironomidae), with description of female, pupa and larva and generis diagnosis for Riethia. Aquatic Insects, 31(4), 247-259.

Villamarín, C. (2012). Estructura y composición de las comunidades de macroinvertebvrados acuáticos en ríos altoandinos de Ecuador y Perú. Diseño de un sistema de medida de la calidad del agua con índices multimétricos (Tesis de Doctorado). Universidad de Barcelona, España. Recuperado de http://www.tdx. cat/handle/10803/83923

Villamarin, C., Rieradevall, M., Paul, M. J., Barbour, M. T., \& Prat, N. (2013) A tool to assess the ecological condition of tropical high Andean streams in Ecuador and Peru: The IMEERA index. Ecological Indicators, 29, 79-92.

Weigend, M., Gottschling, M., Hilger, H. H., \& Nück, N. M. (2010). Five new species of Lithospermum L. (Boraginaceae tribe Lithospermeae) in Andean South America: Another radiarion in the Amotape-Huancabamba Zone. Taxon, 59(4), 1161-1179.

Wiedenbrug, S. \& Fittkau, E. J. (1997). Oliveiriella almeidai (Oliveira, 1946), gen nov, comb. Nov, from South America with description of the pupae. Spixiana, 20(2), 167-172.

Wiedenburg, S. \& Ospina-Torres, R. (2005). A key to pupal exuviae of Neotropical Tanytarsinii (Diptera, Chironomidae). Amazoniana, XVIII(3/4), 17-317.

Wiedenbrug, S. \& Trivino-Strixino, S. (2009). Ubatubaneura, a new genus of the Corynoneura group (Diptera Chironomidae: Orthocladiinae) from the Brazilian Atlantic Forest. Zootaxa, 1993, 41-52.

Wiedenbrug, S., Mendes, H. F., Pepinelli, M., \& TrivinoStrixino, S. (2009). Review of the genus Onconeura Anderson \& Saether (Diptera: Chironomidae), with the description of four new species from Brazil. Zootaxa, 2265, 1-26.

Wiedenbrug, S. \& Trivino-Strixino, S. (2011). New species of the genus Corynoneura Winnertz (Diptera, Chironomidae) from Brazil. Zootaxa, 2822, 1-40.

Wiederholm, T. (Ed). (1986). Chironomidae of the Holarctic Region. Keys and diagnoses. Part. 2 Pupae. Entomologica Scandinavica, (Suppl. 28), 482. 